Article

\title{
IgE-Binding Epitopes of Pis v 1, Pis v 2 and Pis v 3, the Pistachio (Pistacia vera) Seed Allergens
}

\author{
Annick Barre ${ }^{1}(0)$, Christophe Nguyen ${ }^{2}$, Claude Granier ${ }^{3}$, Hervé Benoist ${ }^{1}$ and Pierre Rougé ${ }^{1, *}$ \\ 1 UMR 152 PharmaDev, Faculté de Pharmacie, Institut de Recherche et Développement, \\ Université Paul Sabatier, 35 Chemin des Maraîchers, 31062 Toulouse, France; \\ annick.barre@univ-tlse3.fr (A.B.); herve.benoist@ird.fr (H.B.) \\ 2 IBMM CNRS, Faculté de Pharmacie, Institut des Biomolécules Max Mousseron, 15 Avenue Charles Flahault, \\ BP 14491, CEDEX 5, 34093 Montpellier, France; christophe.nguyen@umontpellier.fr \\ 3 Sys2Diag UMR 9005 CNRS/ALCEDIAGComplex System Modeling and Engineering for Diagnosis, \\ Cap delta/Parc Euromédecine, 1682 rue de la Valsière CS 61003, CEDEX 4, 34184 Montpellier, France; \\ claude.granier@sys2diag.cnrs.fr \\ * Correspondence: pierre.rouge.perso@gmail.com; Tel.: +33-06-9552-0851
}

Received: 3 February 2021; Accepted: 19 March 2021; Published: 23 March 2021

\begin{abstract}
Sequential IgE-binding epitopes were identified on the molecular surface of the Pis v 1 (2S albumin), Pis v 2 (11S globulin/legumin) and Pis v 3 (7S globulin/vicilin)—-major allergens from pistachio (Pistacia vera) seeds-using the Spot technique. They essentially consist of hydrophilic and electropositively charged residues well exposed on the surface of the allergens. Most of the epitopic regions identified on Pis v 1 and Pis v 3 do not coincide with the putative $N$-glycosylation sites and thus are not considered as glycotopes. Surface analysis of these epitopic regions indicates a high degree of conformational similarity with the previously identified epitopic regions of the corresponding allergens Ana o 1 (vicilin), Ana o 2 (legumin) and Ana o 3 (2S albumin) from the cashew (Anacardium occidentale) nut. These results offer a molecular basis for the IgE-binding cross-reactivity often observed between pistachio and cashew nut. They support the recommendation for prescribing pistachio avoidance in cashew allergic patients. Other conformational similarities were identified with the corresponding allergens Ses i 1 (2S albumin), Ses i 3 (vicilin) and Ses i 6 (legumin) from sesame (Sesamum indicum), and Jug r 1 (2S albumin), Jug r 2 (vicilin) and Jug r 4 (legumin) from walnut (Juglans regia). Conversely, conformation of most of the epitopic regions of the pistachio allergens often differs from that of epitopes occurring on the molecular surface of the corresponding Ara $\mathrm{h} 1$ (vicilin), Ara h 2 (2S albumin) and Ara h 3 (legumin) allergens from peanut (Arachis hypogaea).
\end{abstract}

Keywords: pistachio; cashew nut; sesame; walnut; allergens; IgE-binding epitopes; IgE-binding cross-reactivity

\section{Introduction}

The IgE-mediated anaphylaxis to tree nuts, such as almond (Prunus dulcis), Brazil nut (Bertholletia excelsa), cashew nut (Anacardium occidentale), hazelnut (Corylus avellana), pecan nut (Carya illinoinensis), pistachio nut (Pistacia vera), walnut (Juglans regia), has now become a public health concern, responsible for a major proportion of often severe anaphylactic shocks in both children and adults [1-3]. Although belonging to different botanical families, tree nuts share with peanut (Arachis hypogaea) and other edible seeds from lentil (Lens culinaris), pea (Pisum sativum), kidney bean (Phaseolus vulgaris), soybean (Glycine max), sesame (Sesamum indicum) and buckwheat (Fagopyrum esculentum), three groups of $2 \mathrm{~S}$ albumins, 7S globulins or vicilins, and 11S globulins or legumins that have been recognized for a long time as being the most frequent allergen sources in seeds [4]. However, except for peanut and 
hazelnut, seed allergens from other tree nuts are less well known. This is the case with pistachio allergens, which are responsible for sometimes severe allergic manifestations. Ingestion of roasted pistachio nuts (Pistacia vera) has been reported to cause potentially harmful anaphylactic reactions $[5,6]$, but pistachio is also currently incorporated in ice creams, bakery products and various exotic dishes. Food-dependent exercise-induced anaphylaxis to pistachio has also been reported [7]. However, anaphylaxis to pistachio is most often associated to cashew anaphylaxis $[8,9]$.

To date, three major pistachio seed storage protein allergens have been identified in pistachio, corresponding to a $2 \mathrm{~S}$ albumin (Pis v 1), a 11S globulin (Pis v 2) and a 7S globulin (Pis v 3) $[10,11]$. Another $11 \mathrm{~S}$ globulin closely related to Pis v 2, Pis v 5, was further reported to occur in pistachio seed [acces. Q8GZP6]. In addition, another major allergen, Pis v 4, which consists of a [Mn]SOD (superoxyde dismutase) unrelated to seed storage proteins, was identified in pistachio [12]. These allergens closely resemble the corresponding allergens from peanut (the $7 \mathrm{~S}$ globulin Ara $\mathrm{h}$ 1, the v2S albumin Ara h 2, and the 11S globulin Ara h 3) [13-15] and other tree nuts, and cross-react, especially, with the 7S globulin Ana o 1, the 11S globulin Ana o 2 and the $2 S$ albumin Ana o 3 of cashew (Anacardium occidentale) [16,17], which belongs to the same botanical family of Anacardiaceae. Additional cross-reactivity with the homologous allergens from more distantly-related seeds, has been also reported [18-22].

The present work was aimed at identifying the IgE-binding epitopes of the major allergens of pistachio, Pis v 1 (2S albumin), Pis v 2 (11S globulin/legumin), and Pis v 3 (7S globulin/vicilin), as the molecular basis for their cross-reactivity with other corresponding tree nut allergens.

\section{Materials and Methods}

\subsection{Plant Material and Human Sera}

Raw seeds from pistachio (Pistacia vera), cashew nut (Anacardium occidentale) and sesame (Sesamum indicum) were freely obtained from SOFICOR-Menguy's company (Toulouse, France).

Immune sera were drawn after informed consent of patients experiencing anaphylaxis to cashew nut (Table 1). Their reactivity towards pistachio was checked in Western blot experiments using a freshly prepared pistachio protein extract in Tris buffered saline ( $\mathrm{pH} 7.5)$ as an allergen source. The specificity of the used sera had been assessed in previous publications $[8,23]$.

Table 1. List of sera from subjects allergic to cashew nut and pistachio.

\begin{tabular}{cccc}
\hline Subjects & Sex/Age (Years) & $* *$ Pistachio Specific IgE (KU/L) & $* * *$ Cashew Specific IgE (KU/L) \\
\hline 1 & $* \mathrm{M} / 14$ & $>100$ & 83.8 \\
2 & $\mathrm{M} / 5$ & 2.20 & 1.35 \\
3 & $\mathrm{M} / 8$ & 0.36 & 0.25 \\
4 & $\mathrm{M} / 4$ & 0.17 & 0.45 \\
5 & $\mathrm{M} / 9$ & 1.65 & 1.51 \\
6 & $\mathrm{M} / 16$ & 0.47 & 0.10 \\
7 & ${ }^{*} \mathrm{~F} / 7$ & 5.69 & 3.92 \\
8 & $\mathrm{~F} / 8$ & 2.54 & 1.44 \\
9 & $\mathrm{M} / 4$ & 2.46 & 1.20 \\
\hline
\end{tabular}

${ }^{*}$ M: male, F: female, ${ }^{* *}$ ImmunoCAP Phadia F203, ${ }^{* * *}$ ImmunoCAP Phadia F202.

Sera from patients allergic to cashew were used as probes to identify the IgE-binding cross-reacting epitopic stretches from Pis v 1, Pis v 2, Pis v 3 and Ana o 3, immobilized on cellulose sheets. Sera from patients allergic to sesame (Table 2), were used as probes to identify the IgE-binding epitopic stretches from Ses i 3 and Ses i 6, immobilized on cellulose sheets. 
Table 2. List of sera from subjects allergic to sesame.

\begin{tabular}{ccc}
\hline Subjects & Sex/Age (Years) & * Sesame Specific Ige (KU/L) \\
\hline 1 & $\mathrm{M} / 6$ & 0.10 \\
2 & $\mathrm{M} / 16$ & 0.26 \\
3 & $\mathrm{~F} / 10$ & 0.74 \\
4 & $\mathrm{M} / 10$ & 0.26 \\
5 & $\mathrm{M} / 5$ & 1.53 \\
6 & $\mathrm{M} / 10$ & 1.85 \\
7 & $\mathrm{M} / 10$ & 14.2 \\
8 & $\mathrm{~F} / 6$ & 46.3 \\
9 & $\mathrm{~F} / 9$ & 84.4 \\
\hline
\end{tabular}

* ImmunoCAP Phadia F10.

\subsection{Analytical Methods}

Protein extract was prepared from frozen seeds by two grinding steps of $40 \mathrm{~s}$ each in a Fast Prep-24 homogenizer (MP Biomedicals, Illkirch, France), in $20 \mathrm{mM}$ Tris- $\mathrm{HCl}$ buffered saline (pH 7.4). The resulting slurry was centrifuged at $15,000 \times g$ for $10 \mathrm{~min}$ at $4{ }^{\circ} \mathrm{C}$. The supernatant fraction was carefully collected while avoiding the floating lipid layer, filtered through a $0.2 \mu \mathrm{m}$ membrane, and stored at $-20{ }^{\circ} \mathrm{C}$ until used.

The protein content of the pistachio extract was estimated using the bicinchonic acid kit reagent (Pierce) [24] with bovine serum albumin as a standard. The protein extract was checked by SDS-PAGE in $15 \%$ polyacrylamide gels using Tris-glycine as trailing ion [25] and staining with Coomassie blue. Coomassie blue stained bands were digested with trypsin in the gel and mass mapped by MALDI-TOF analysis as previously described [26]. The software Protein Prospector was used for the identification of the protein using the NCBI non-redundant database.

\subsection{Immunoblotting}

IgE-containing sera from allergic patients were used in Western blotting as probes for the pistachio allergens. Following 1D SDS-PAGE, proteins were transferred onto a Protran nitrocellulose $0.2 \mu \mathrm{m}$ membrane (Sigma-Aldrich, L'Isle d'Abeau Chesnes, France) at $20 \mathrm{~V}$ during $45 \mathrm{mn}$ using a $48 \mathrm{mM}$ Tris/39 mM glycine/20\% (v/v) methanol mixture. After an overnight incubation in $10 \mathrm{mM}$ PBS (pH 7.4) containing $0.2 \%(v / v)$ tween and $5 \%(v / v)$ skimmed milk, the membrane was soaked in the patient IgE-containing sera diluted 1:10 in the same buffer and incubated for $2 \mathrm{~h}$ in a moist chamber. After three washings of $10 \mathrm{~min}$ each with the same buffer, the membrane was soaked in rabbit HRP-labelled anti-human IgE diluted 1/5000 in the buffer and incubated for $1 \mathrm{~h}$ under gentle stirring. Following three washings of $10 \mathrm{~min}$ each with buffer, the immunolabelled spots were detected using the ECL Plus detection (ThermoScientific, Illkirch, France) after 3 min exposure in cassette. All the handling was carried out at room temperature.

\subsection{Peptide Synthesis and IgE-Binding Epitope Mapping}

Overlapping 15-mer peptides, frameshifted by three residues, covering the entire amino acid sequences of Pis v 1, Pis v 2 and Pis v 3 were prepared by using the SPOT technique [27]. The protocol previously described in detail [28] was followed with the exception of the utilization of the Multipep automatic Spot synthesizer (Intavis). Briefly, peptides were assembled using the Fmoc chemistry on a cellulose membrane harboring an amino polyethylene glycol moiety. The $C$-terminal residue of each peptide was coupled to the activated membrane. After Fmoc deprotection, the following amino acids were sequentially added. At the end of the synthesis, side chain protecting groups were removed by a trifluoracetic acid treatment while the linkage of the peptides to the membrane was maintained.

The membrane was soaked overnight into $20 \mathrm{~mL}$ of Tris-buffered saline (TBS) containing $2 \mathrm{~mL}$ blocking buffer (ThermoFisher, Montigny-le-Bretonneux, France) and $1 \mathrm{~g}$ sucrose (pH 7.0), and then washed three times with TBS containing $0.1 \%(v / v)$ tween (TBSTw). A 1:10 $(v / v)$ diluted pool of patient 
sera was added in the presence of an anti-protease cocktail Roche (Sigma-Aldrich, L'Isle d'Abeau Chesnes, France) and the membrane was incubated in a moist chamber for $2 \mathrm{~h}$. After three washes with TBSTw (pH 7.0) the membrane was stirred in a 1:4000 dilution of mouse monoclonal anti-human IgE labelled with alkaline phosphatase (Sigma-Aldrich, L'Isle d'Abeau Chesnes, France) for $1 \mathrm{~h}$. After three washes with TBSTw ( $\mathrm{pH}$ 7.0), the interacting peptide spots were colored for $30 \mathrm{~min}$ by adding the 5-bromo-4-chloro-3-indolylphosphate (BCIP) substrate for alkaline phosphate (Promega). The membrane was washed three times with deionized water and dried for scanning. The membrane can be used repeatedly after a regeneration step consisting in one wash in dimethylformamide for 1 $\mathrm{min}$, three washes in deionized water, three washes in $8 \mathrm{M}$ urea containing $1 \%(w / v)$ SDS and $1 \%(w / v)$ b-mercaptoethanol and three subsequent washes in a mixture of ethanol-acetic acid-water (50:10:40, $v / v / v)$. A similar protocol was used to map IgE-binding epitopes along the amino acid sequence of Ana o 3 (2S albumin) from Anacardium occidentale, and Ses i 3 (7S globulin/vicilin) and Ses i 6 (11S globulin/legumin) from sesame (Sesamum indicum) seed.

\subsection{Bioinformatics}

The amino acid sequences of Pis v 1 (acces. ABG73108.1), Pis v 2 (acces. ABU42022.1) and Pis v 3 (ABO36677.1) were taken from the NCBI protein database (http://www.ncbi.nlm.nih.gov/pubmed, accessed on 7 January 2021). The multiple amino acid sequence alignment was performed with MacVector, using the T-coffee program [29]. Unrooted phylogenetic trees were built up from the multiple alignment of $2 \mathrm{~S}$ albumins, vicilins (7S globulins) and legumins (11S globulins), using the uncorrected neighbor joining method integrated in MacVector.

Homology modelling of Pis v 1, Pis v 2, Pis v 3, Ana o 3 and Ses i 3 was performed with YASARA [30], using appropriate templates from the Protein Data Bank (PDB) [31]. Five different models were built for Pis v 1, using 2S albumin from Moringa oleifera (PDB code 5DOM) [32], Ara h 2 from Arachis hypogaea (PDB code 3OB4) [33], Ber e 1 from the Brazil nut Bertholletia excels (PDB code 2LVF) [34], the seed storage albumin from Helianthus annuus (PDB code 5U87) (to be published), and the sweet protein mabinlin II from Capparis masaikai (PDB code 2DS2) [35], as appropriate templates. Nine different models were built for Pis v 3 (vicilin, 7S globulin) using vicilin from eggplant Solanum melongena (PDB code 5CAD, 5VF5) [36,37], vicilin-like protein from Capsicum annuum (PDB code 5YJS) [38], Car i 2 (vicilin) from the pecan nut Carya illinoinensis (PDB code 5E1R) [39], and vicilin from Pinus koraiensis (PDB code 4LEJ) [40], as appropriate templates. Nineteen different models were built for Pis v 2 (legumin, 11S globulin) using 11S globulin from Wrightia tinctoria (PDB code 5WXU) [41], cocosin (legumin) from the coconut Cocos nucifera (PDB code 5WPW) [42], subunit $\beta$ of 11 S globulin from Cucurbita maxima (PDB code 2E9Q) [43], 11S globulin from Amaranthus hypochondriacus (PDB code 3QAC) (to be published), and prunin-1 (11S globulin) from almond Prunus dulcis (PDB code 3FZ3) [44], as appropriate templates. Five models were built for Ana o 3 (acces. AAL91665.1), using Ara h 2 from Arachis hypogaea (PDB code 3OB4) [32], mabinlin II from Capparis masaikai (PDB code 2DS2) [35], $0.19 \alpha$-amylase inhibitor from wheat Triticum aestivum (PDB code 1HSS) [45], and Ara h 6 (conglutin) from peanut Arachis hypogaea (PDB code 1W2Q) [46], as appropriate templates. Ten different models were built for Ses i 3 (acces. AAK15089.1), using the same templates as those selected for Pis v 3 [36-40]. Twenty-five different models were built for Ses i 6 (AAD42944.1), using five different templates 5WXU [40], 5WPW [41], 2E9Q [42], 3FZ3 [43], and Ara h 3 from Arachis hypogaea (PDB code 3C3V) [47]. Finally, a single hybrid model was built up from the different previous models, for each of the modelled proteins. PROCHECK [48], ANOLEA [49], and the calculated QMEAN scores [50,51], were used to assess the geometric and thermodynamic qualities of the three-dimensional models (Table 3). Molecular surfaces and Coulombic charges (negative, positive and neutral surfaces colored red, blue and white, respectively) were calculated and rendered with Chimera [52]. 
Table 3. Geometric and thermodynamic qualities of the three-dimensional models built by homology modeling with YASARA.

\begin{tabular}{cccccc}
\hline Models & $\begin{array}{c}\text { 3D Structures from the } \\
\text { PDb Used as Templates }\end{array}$ & $\begin{array}{c}\text { Number of Models } \\
\text { Built by Homology } \\
\text { Modelling }\end{array}$ & $\begin{array}{c}\text { Residues out of the } \\
\text { Allowed Areas in the } \\
\text { Ramachandran Plot }\end{array}$ & $\begin{array}{c}\text { * Residues with Values } \\
\text { over the Threshold in } \\
\text { ANOLEA Plot }\end{array}$ & $\begin{array}{c}\text { QMEAN } \\
\text { Value }\end{array}$ \\
\hline Pis v 1 & $\begin{array}{c}\text { 2D2S, 2LVF, 3OB4, 5DOM, } \\
\text { 5U87 }\end{array}$ & 5 & 0 & 20 over 122 residues & -1.19 \\
Pis v 2 & $\begin{array}{c}\text { 2E9Q, 3QAC, 3FZ3, } \\
\text { 5WPW, 5WXU }\end{array}$ & 19 & 3:S201(A),D249(A),D249(B) & 27 over 440 residues & -1.78 \\
Pis v 3 & 4LEJ, 5CAD, 5E1R, 5VF5, & 9 & 2:K334(B),F502(C) & 23 over 405 residues & -0.21 \\
Ana o 3 & 1HSS, 1W2Q, 2DS2, 3OB4 & 5 & 0 & 20 over 114 residues & -1.55 \\
Ses i 3 & 4LEJ, 5CAD, 5E1R, 5VF5, & 10 & 0 & 21 over 406 residues & -0.39 \\
5es i 6 & 2E9Q 3ZF3, 5WPW, & 25 & 3:Q121(A),R234(C),S248(C) & 29 over 438 residues & -1.08 \\
\hline
\end{tabular}

The number of residues $\left(^{*}\right)$ refer to those occurring in the different model monomers.

\section{Results}

\subsection{The Pistachio Allergens}

To date, five allergens from pistachio seeds have been identified and characterized, two major allergens Pis v 1 (2S albumin) [10] and Pis v 2 (11S globulin/legumin), and three additional allergens Pis v 5 (11S globulins, legumin) [acces. B7SLJ1], Pis v 3 (7S globulin, vicilin) [11] and Pis v 4 (SOD) [12], as mentioned in the WHO/IUIS Allergen Nomenclature database (http://www.allergen.org, accessed on 9 January 2021) [53]. In addition, another vicilin-like, two additional 11S globulins and oleosins, have been identified from the genome assembly available for pistachio (GCF_008641045.1) but we do not know whether the identified potential allergens are actually expressed in pistachio seeds.

The pistachio allergens Pis v 1, Pis v 2 and Pis v 5, and Pis v 3, expressed in mature pistachio seeds, accumulate gradually in protein bodies during the seed ripening process, as seed storage proteins (Figure 1A). Major allergens of protein bodies are easily detected in immunofluorescence experiments, using sera from patients allergic to cashew as primary antibody and an anti-human secondary antibody coupled to Alexa 633, respectively (Figure 1B).

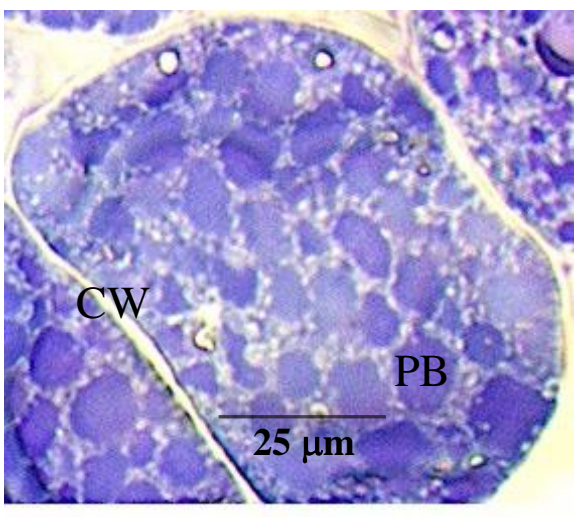

(A)

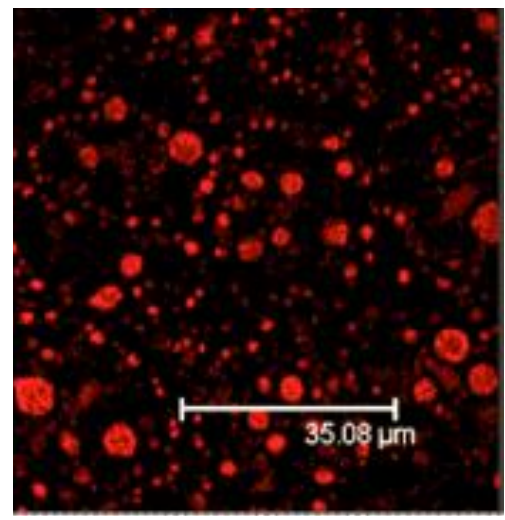

(B)

Figure 1. (A) Toluidine blue-staining of seed storage proteins located into the protein bodies (PB) of cotyledon cells of pistachio. CW: cell wall. Tiny white globules arrayed in the cytoplasm correspond to oleosin-containing oil bodies. (B) Binding of IgE from patient sera allergic to cashew nut and pistachio, to the major allergen-containg protein bodies of cotyledon cells (red spots). Alexa-labelled anti-IgE was used as probe to reveal the binding of IgE-containing sera from cashew allergic patient to protein bodies.

Upon SDS-PAGE, the major allergens from pistachio are well separated and readily interact with the IgE-containing patient sera in Western blot experiments (Figure 2). However, depending on the patient sera, different allergens are predominantly recognized by the IgE antibodies. Accordingly, 
several different patient sera should be used to accurately identified the IgE-binding epitopic stretches of Pis $\mathrm{v} 1$, Pis $\vee 2$ and Pis $\vee 3$ mapped on the corresponding activated membranes.

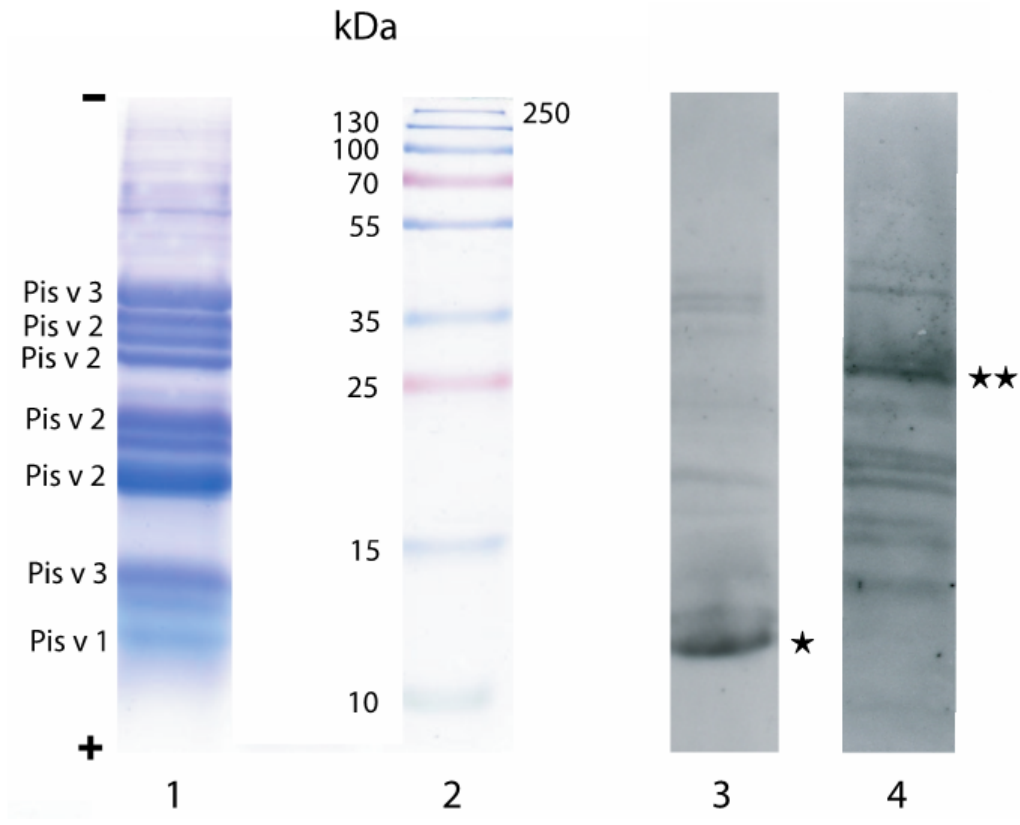

Figure 2. SDS-PAGE of a protein extract from pistachio nut (line 1) and Western blots of allergic patient sera that preferentially react with Pis v 1 (line 3) and Pis v 2 (lane 4). Molecular weight markers are indicted on lane 2. Protein fractions corresponding to the Pis v 1, Pis v 2 and Pis v 3 allergens were identified by MALDI-TOF after trypsic digestion in gel of the protein bands (line 1 ). ${ }^{*}$ and ${ }^{* *}$ indicate the most IgE-reactive protein fractions.

\subsection{Pis $v 1$ and Closely Related $2 S$ Albumins}

Homology modelling of the Pis $v 1$ allergen from appropriate templates yielded a typical $2 \mathrm{~S}$ albumin structure built up of two $\alpha$-helix-containing polypeptide chains linked together by two conserved disulfide bridges (Figure 3C,F). As predicted from the GlyProt server (http://www.glycosciences.de/ modeling/glyprot/php/main.php, accessed on 9 January 2021) [54], the $N$-terminal putative $N$-glycan site at 13NLS should be actually glycosylated.

Four main IgE-binding epitopic stretches were identified along the amino acid sequence of Pis $\mathrm{v} 1$ in Spot experiments using sera that predominantly recognized Pis v 1 in Western blots as a probe (Figure 3A,B). These 3 stretches correspond to four distinct IgE-binding regions exposed on the molecular surface (Figure 3D,G). Most of these epitopes contain charged residues and coincide with the electropositively and electronegatively charged regions occurring at the molecular surface of the allergen (Figure 3E,H).

The modelled $2 S$ albumin allergen from cashew nut, Ana o 3, exhibit a very similar fold (Figure 4C,F), and four main IgE-binding epitopic stretches were similarly identified along the amino acid sequence of Ana o 3 (Figure 4D,G), which also correspond to electropositively and electronegatively charged regions occurring on the molecular surface of the allergen (Figure $4 \mathrm{E}, \mathrm{H}$ ). Very similar pictures were previously obtained with other $2 \mathrm{~S}$ albumin allergen Jug $\mathrm{r} 1$ from walnut (Juglans regia) [55] and Ses i 1 from sesame (Sesamum indicum) [56]. 
A

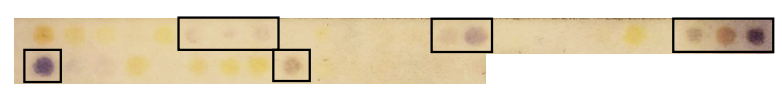

B

SIYRATVEVEGENLSSGOSCQKOFEEQOKFKHCQMYVQQEVQKSODG

HSLTARINQRQQCFKQCCOELOEVDKKCRCONLEOMVKROQQQGQFR GEKLQELYETASELPRMCNISPSQGCQFSSPYWSY
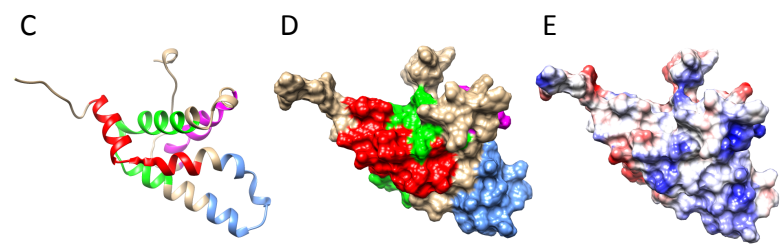

$\mathrm{F}$

G

$\mathrm{H}$

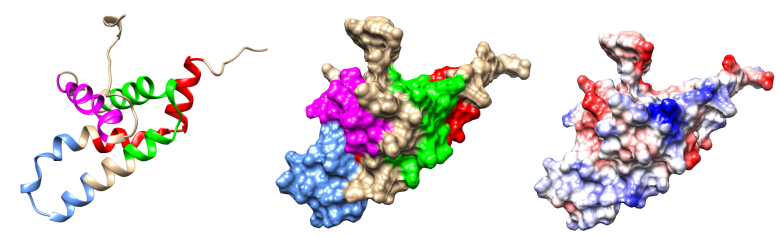

Figure 3. (A) IgE-binding peptides (boxed violet spots) revealed on the Pis v 1 Spot membrane. (B) Mapping of the corresponding continuous IgE-binding epitopic regions (colored boxed white letters) along the amino acid sequence of Pis v 1. Epitopes are colored red (epitope 1), blue (epitope 2), green (epitope 3) and magenta (epitope 4), respectively. (C,F) Ribbon diagram of the front (C) and back (F) face of the modelled Pis v 1 showing the localization of the IgE-binding epitopic regions numbered and colored as in (B). (D,G) Surfaces occupied by the colored IgE-binding epitopes on the front (D) and back (G) face of Pis v 1 numbered and colored as in (B). (E,H) Mapping of Coulombic charges on the front $(\mathbf{E})$ and back $(\mathbf{H})$ face of Pis $v 1$. Electropositive and electronegative regions are colored blue and red, respectively. Neutral regions are white.
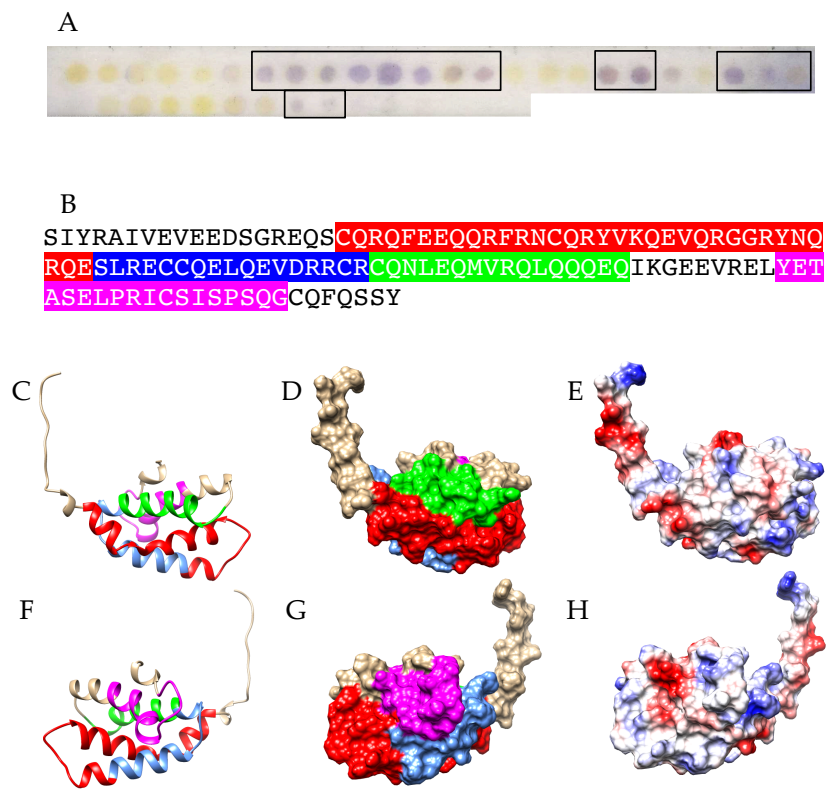

Figure 4. (A) IgE-binding peptides (boxed violet spots) revealed on the Ana o 3 Spot membrane. (B) Mapping of the corresponding continuous IgE-binding epitopic regions (colored boxed white 
letters) along the amino acid sequence of Ana o 3. Epitopes are colored red (epitope 1), blue (epitope 2), green (epitope 3) and magenta (epitope 4), respectively. (C,F) Ribbon diagram of the front (C) and back (F) face of the modelled Ana o 3 showing the localization of the IgE-binding epitopic regions numbered and colored as in (B). (D,G) Surfaces occupied by the IgE-binding epitopes on the front (D) and back (G) face of Ana o 3 numbered and colored as in (B). (E,H). Mapping of Coulombic charges on the front $(\mathbf{E})$ and back $(\mathbf{H})$ of Ana o 3. Electropositive and electronegative regions are colored blue and red, respectively. Neutral regions are white.

Obviously, Pis v 1 and other $2 S$ albumin allergens exhibit a quite superposable three-dimensional fold core, even though their amino acid sequences share rather low percentages of both identity and similarity (Figure 5). However, Pis v 1, Ana o 3 and Ses i 2, are readily clustered in the unrooted phylogenetic tree built for $2 S$ albumin allergens (Figure 6), that suggest strong phylogenetic affinities between these proteins. In this respect, it is noteworthy that Pis v 1 and Ana o 3 belong to the same Anacardiaceae family.

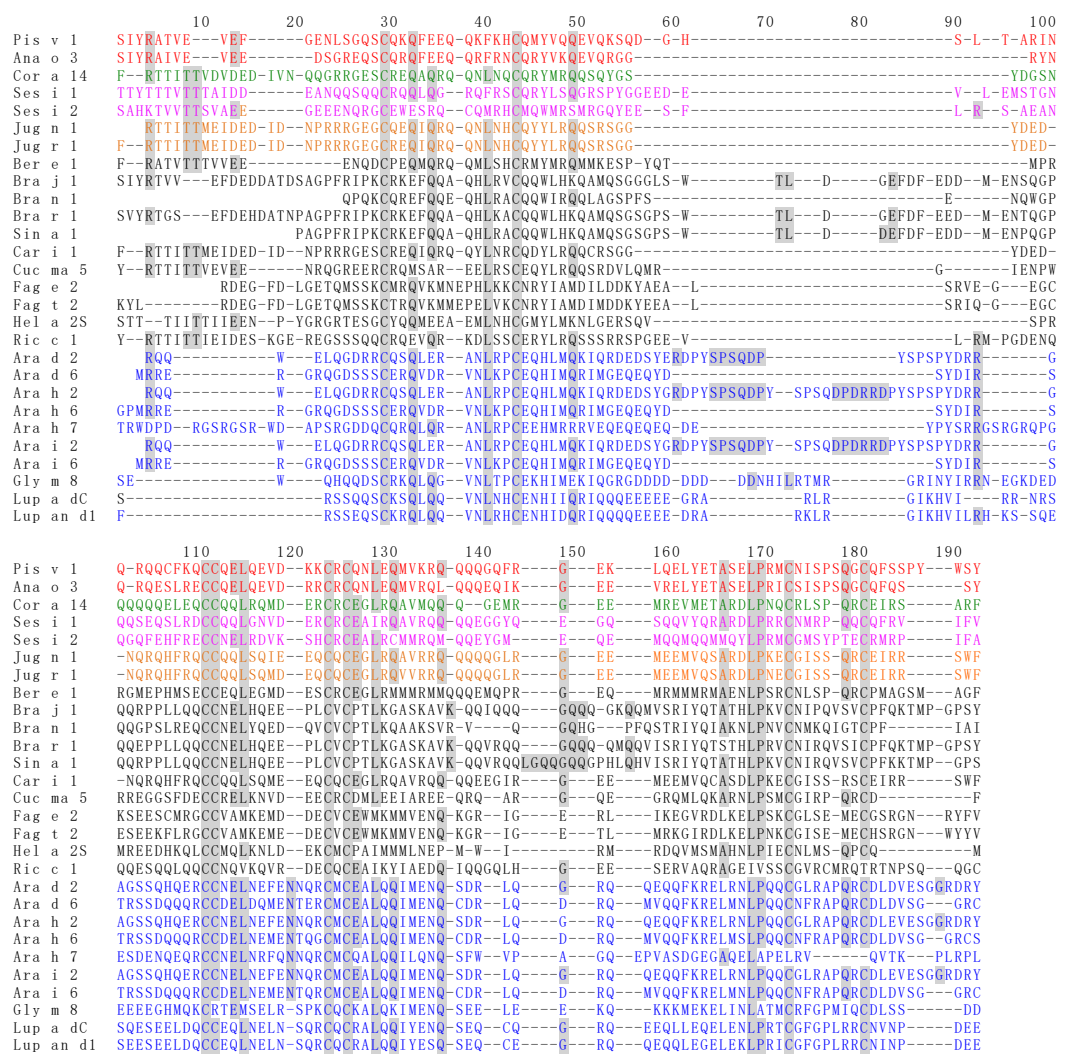

Figure 5. Multiple amino acid sequence alignment of $2 S$ albumin allergens Ana o 3 from Anacardium occidentale; Ara d 2 and Ara d 6 from Arachis duranensis; Ara h 2, Ara h 6 and Ara h 7 from Arachis hypogaea; Ara i 2 and Ara i 6 from Arachis ipaensis; Ber e 1 from Bertholletia excelsa; Bra j 1 from Brassica juncea; Bra $\mathrm{n} 1$ from Brassica napus; Bra $\mathrm{r}$ from Brassica rapa; Car i 1 from Carya illinoinensis; Cor a 14 from Corylus avellana; Cuc ma 5 from Cucurbita maxima; Fag e 2 from Fagopyrum esculentum; Fag $\mathrm{t} 2$ from Fagopyrum tataricum; Gly m 8 from Glycine max; Hel a 2S from Helianthus annuus; Jug $\mathrm{n} 1$ from Juglans nigra; Jug $\mathrm{r} 1$ from Juglans regia; Lup a $\delta \mathrm{C}$ from Lupinus albus; Lup an $\delta 1$ from Lupinus angustifolius; Ric c 1 from Ricinus communis; Ses 11 and Ses i 2 from Sesamum indicum; Sin a 1 from Sinapis alba. Identical amino acids are grey shadded. Aminoacid sequences of allergens belonging to the same botanical family, are similarly colored in red (Anacardiaceae), green (Betulaceae), magenta (Pedaliaceae), orange (Juglandaceae) and blue (Fabaceae). 


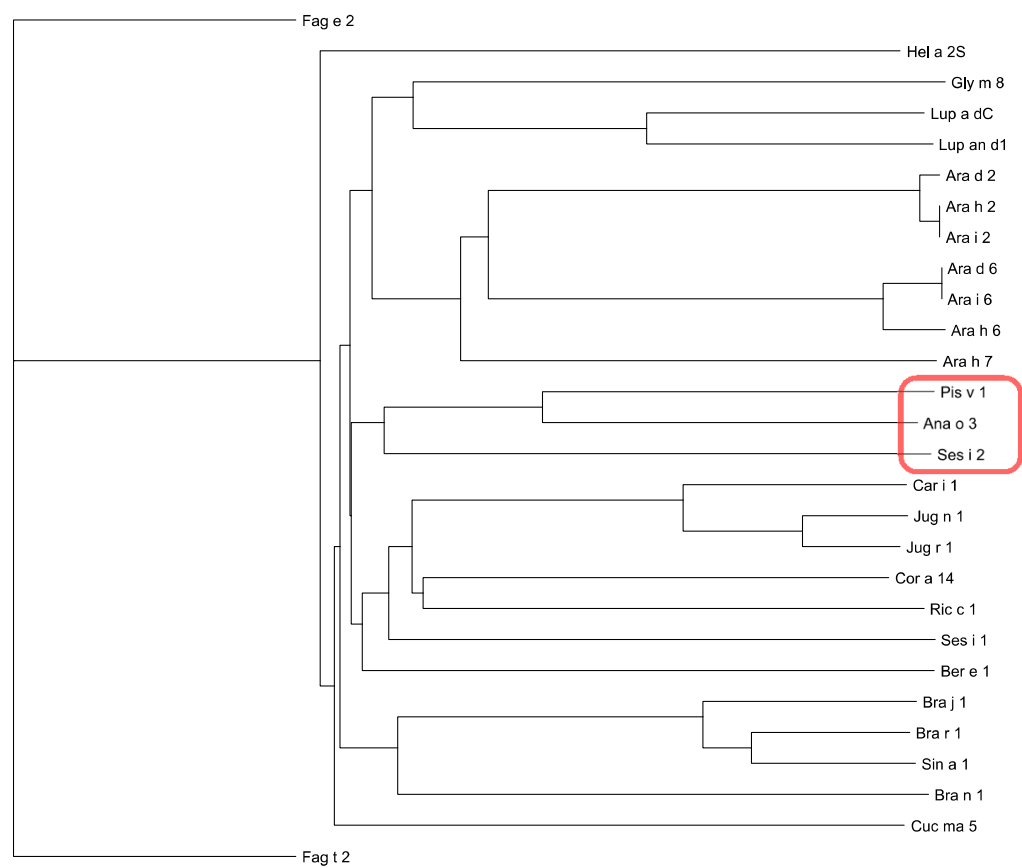

Figure 6. Dendrogram of $2 \mathrm{~S}$ albumins from Brazil nut (Ber e 1 from Bertholletia excelsia), buckwheat (Fag e 2 from Fagopyrum esculentum, Fag t 2 from F. tartaricum), cashew nut (Ana o 3 from Anacardium occidentale), Castor bean (Ric c 1 from Ricinus communis), hazelnut (Cor a 14 from Corylus avellana), lupine (convicilin Lup a $\delta \mathrm{C}$ from Lupinus albus, convicilin Lup an $\delta 1$ from Lupinus annuus), mustard (Sin a 1 from Sinapis alba), peanut (Ara d 2 and Ara d 6 fom Arachis duranensis; Ara h 2, Ara h 6 and Ara h 7 from A. hypogaea; Ara i 2 and Ara i 6 from A. ipaensis), pecan nut (Car i 1 from Carya illinoinensis), pistachio (Pis v 1 from Pistacia vera), pumpkin (Cuc ma 5 from Cucurbita maxima), sesamum (Ses i 1 and Ses i 2 from Sesamum indicum), soybean (Gly $\mathrm{m} 8$ from Glycine max), sunflower (Hel an 2S from Helianthus annuus), turnip and rapeseed (Bra 1 from Brassica juncea and Bra $\mathrm{r} 1$ from B. rapa; Bra $\mathrm{n} 1$ from B. napus), walnut (Jug n 1 from Juglans nigra and Jug $r 1$ from J. regia). 2S-albumins clustered to Pis $\mathrm{v} 1$ are red circled.

The phylogenetic relationships observed between Pis v 1 and Ana o 3 are reflected in the high similarity of their IgE-binding epitopic stretches along their amino acid sequences (Figure 7), which most probably account for the IgE-binding cross-reactivity occurring beetwen Pis v 1 and Ana o 3 .
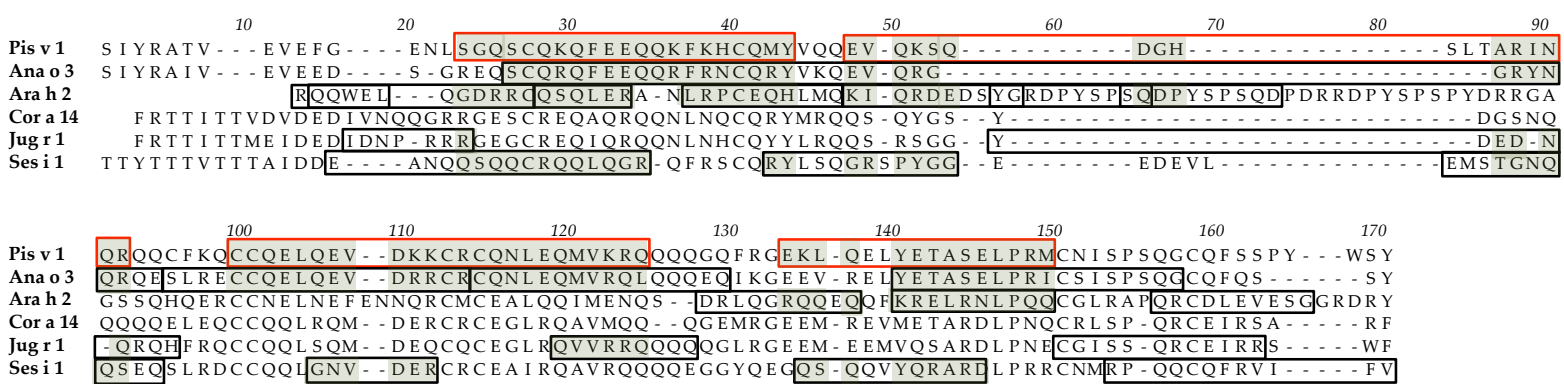

Figure 7. Multiple alignment of $2 \mathrm{~S}$ albumin allergens Pis v 1, Ana o 3, Ara h 2, Cor a 14, Jug r 1 and Ses i 1. The Ig-E-binding epitopes delineated along the amino acid sequence of Pis v 1 (red boxes) and Ana o 3, Ara h 2, Cor a 14, Jug $\mathrm{r} 1$ and Ses 11 (black boxes) are indicated. Amino acid residues occurring in regions of $2 \mathrm{~S}$ albumins homologous to the IgE-binding epitopes of Pis v 3, are shaded pale green.

Other less phylogenetically closely related $2 \mathrm{~S}$ albumin allergens, such as Ses i 1 and Ara h 2, share much less similarity with Pis v 1 and Ana o 3. 


\subsection{Pis $v 3$ and Closely Related $7 S$ Globulins (Vicilins)}

The modelled vicilin allergen Pis $v 3$ corresponds to a homotrimer built from the tail to tail non covalent association of three identical single-chain protomers made of a core of two cupin motifs, extended at both ends by two side arms made up of $\alpha$-helices (Figure 8C,F). Each protomer contains a putative $\mathrm{N}$-glycosylation site at $243 \mathrm{NIT}$, which is predicted to be actually glycosylated according the GlyProt server (http://www.glycosciences.de/modeling/glyprot/php/main.php, accessed on 9 January 2021) [54]. This type of structural organization currently occurs in many other $7 S$ globulin/vicilin allergens from other tree nuts and legume allergens [57].

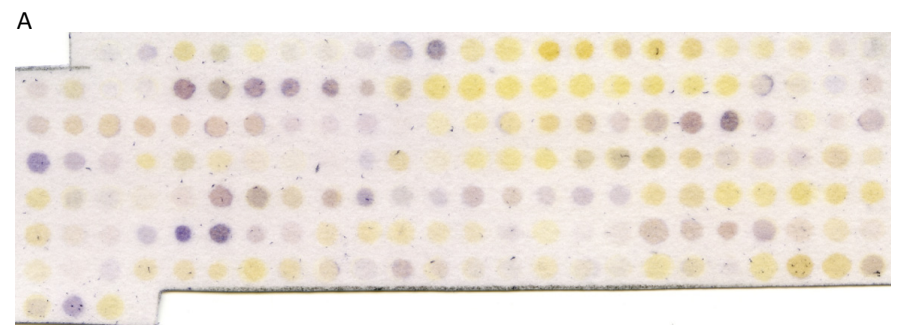

B

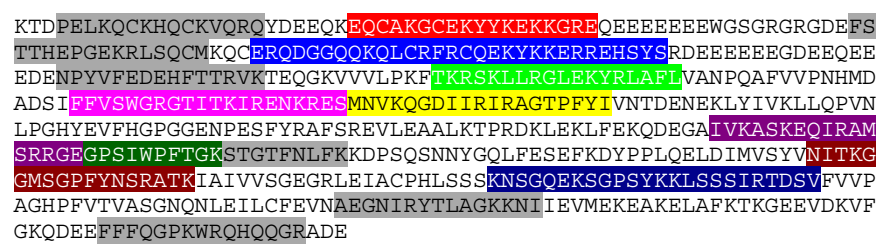

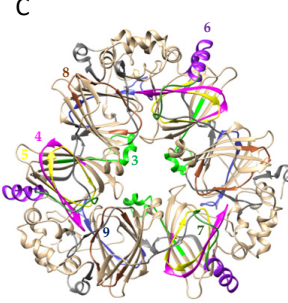

$\mathrm{F}$

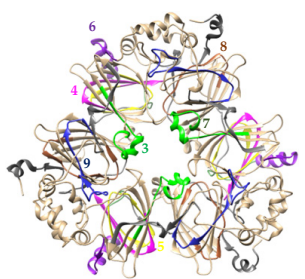

D

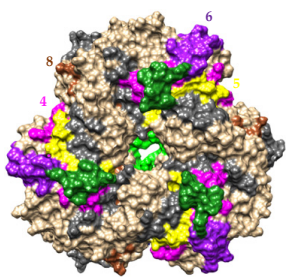

G

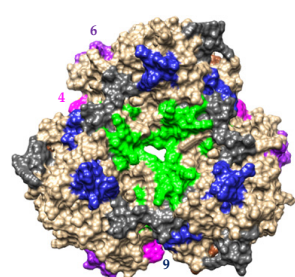

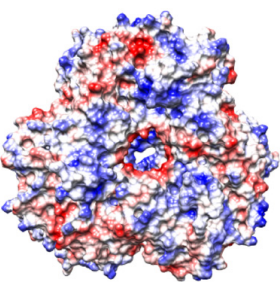

H

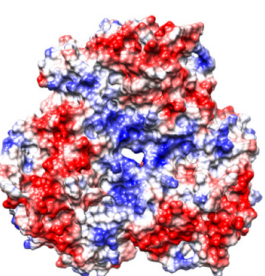

Figure 8. (A) IgE-binding peptides (boxed violet spots) revealed on the Pis v 3 Spot membrane. (B) Mapping of the corresponding continuous IgE-binding epitopic regions (colored boxed white letters) along the amino acid sequence of Ana o 3. Epitopes are colored red (epitope \#1), blue (epitope \#2), green (epitope \#3), magenta (epitope \#4), yellow (epitope \#5), purple (epitope \#6), deep green (epitope \#7), sienna (epitope \#8) and dark blue (epitope \#9), respectively, and numbered as in B. Other single-spot IgE-binding peptides are colored gray. (C,F) Ribbon diagram of the front $(\mathbf{C})$ and back (F) face of the modelled Ana o 3 showing the localization of the colored IgE-binding epitopic regions numbered and colored as in B. IgE-binding epitopic patches corresponding to single-spot IgE-binding peptides, are colored grey. $(\mathbf{D}, \mathbf{G})$ Surfaces occupied by the colored IgE-binding epitopes on the front (D) and back (G) face of Pis v 3. Epitopes are numbered as in (B). (E,H) Mapping of Coulombic charges on the front $(\mathrm{E})$ and back $(\mathrm{H})$ of Pis $\mathrm{v} 3$. Electropositive and electronegative regions are colored blue and red, respectively. Neutral regions are white. 
Up to nine main IgE-binding stretches identified along the amino acid sequence of the Pis $\mathrm{v}$ 3 protomer using the Spot method (Figure $8 \mathrm{~A}, \mathrm{~B}$ ), correspond to more or less exposed IgE-binding epitopic areas arrayed on both faces of the Pis $v 3$ homotrimer (Figure $4 \mathrm{C}, \mathrm{F}$ ). In fact, no information is available on the exposition of epitopes \#1 and \#2 at the surface of Pis $\mathrm{v} 3$ since both epitopes occur in the $\mathrm{N}$-terminal region of the polypeptide chain which is lacking in the three-dimensional model built for Pis $\mathrm{v} 3$ by homology modelling. Two other epitopic region corresponding to epitopes \#4 (colored magenta) and \#8 (colored sienna), are almost completely buried and very little exposed on the surface of the allergen (Figure 4D,G). Like for other pistachio allergens, the well exposed epitopic regions corresponding to epitopes \#3, \#5, \#6, \#7 and \#9, respectively, mostly coincide with both electronegatively (colored red) and electropositively (colored blue) charged regions, and their coalescence creates more extended epitopic regions on the molecular surface of the allergen. Other single surface-restricted epitopes (colored medium blue) are usually well exposed and mostly coalescent with the more extended IgE-binding patches. In addition, the exposed region of epitope \#7 (colored sienna) could correspond to a CCD since it contains the putative 367NIT N-glycosylation site occurring on the amino acid sequence of Pis v 2.

Up to twelve IgE-binding stretches were similarly identified along the amino acid sequence of the corresponding protomer Ses i 3 from sesame seeds, using the Spot method (Figure 9A,B) and, similarly, some epitopic regions corresponding to epitopes \#4 (colored magenta) and \#6 (colored purple), are poorly exposed at the surface of Ses i 3 whereas other exposed epitopes \#2, \#3, \#5, \#7, \#8, \#9, \#10, \#11 and \#12 (Figure 9C,D,F,G), readily coincide with electronegatively (colored red) and electropositively (colored blue) charged regions arrayed on the molecular surface of Ses i 3 (Figure 9E,H).

A

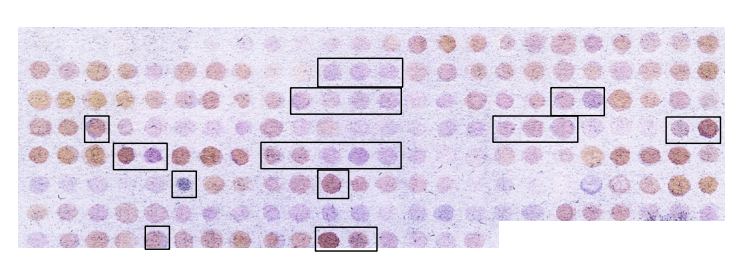

B

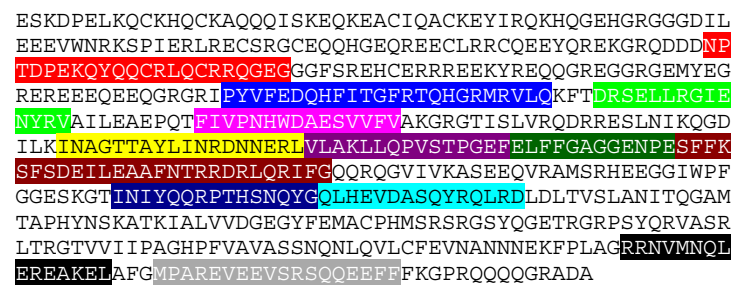
LTRGTVVI I PAGHPFVAVASSNQNLQVLCFEVNANNNEKFPLAG
EREAKELAFGMPAREVEEVSRSQQEEFEFKGPRQQQQGRADA

C
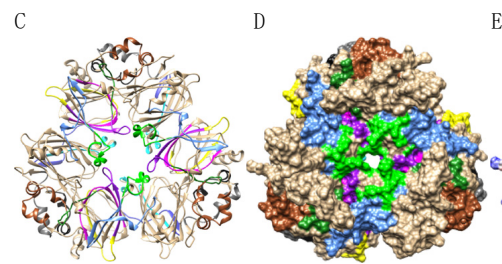

D
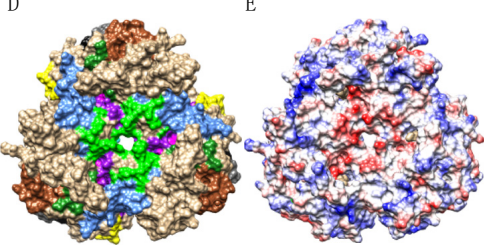

G

H

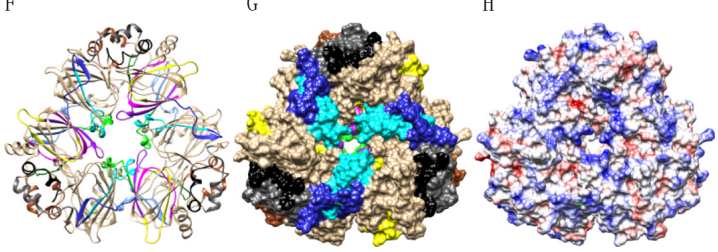

Figure 9. (A) IgE-binding peptides (boxed violet spots) revealed on the Ses i 3 Spot membrane. (B) Mapping of the corresponding continuous IgE-binding epitopic regions (colored boxed white 
letters) along the amino acid sequence of Ana o 3. Epitopes are colored red (epitope \#1), blue (epitope \#2), green (epitope \#3), magenta (epitope \#4), yellow (epitope \#5), purple (epitope \#6), deep green (epitope \#7), sienna (epitope \#8), deep blue (epitope \#9), cyan (epitope \#10), black (epitope \#11) and grey (epitope \#12), respectively. (C,F) Ribbon diagram of the front (C) and back (F) face of the modelled Ses i 3 homotrimer, showing the localization of the colored IgE-binding epitopic regions. Epitopes are numbered and colored as in (B). (D,G) Surfaces occupied by the colored IgE-binding epitopes on the front (D) and back (G) face of Ses i 3. Epitopes are numbered and colored as in (B). (E,H) Mapping of Coulombic charges on the front $(\mathbf{E})$ and back $(\mathbf{H})$ face of Ses i 3. Electropositive and electronegative regions are colored blue and red, respectively. Neutral regions are white.

According to the common and superposable fold of the 7S globulin/vicilin allergens together with their epitopic similarities, the multiple alignment of these seed allergens exhibits a high degree of both identity and similarities, especially for Pis v 3 and Ana o 1, which belong to the same family of Anacardiaceae, and to a lesser extent, for Cor a 11 and Jug r 6 (Juglandaceae) and Ses i 3 (Pedaliaceae) (Figure 10). Accordingly, Pis v 3 and Ana o 1, and Cor a 11, Jug r 6 and Ses i 3, are distributed in two phylogenetically closely related clusters in the unrooted phylogenetic tree built for the $7 \mathrm{~S}$ globulin/vicilin allergens (Figure 11).

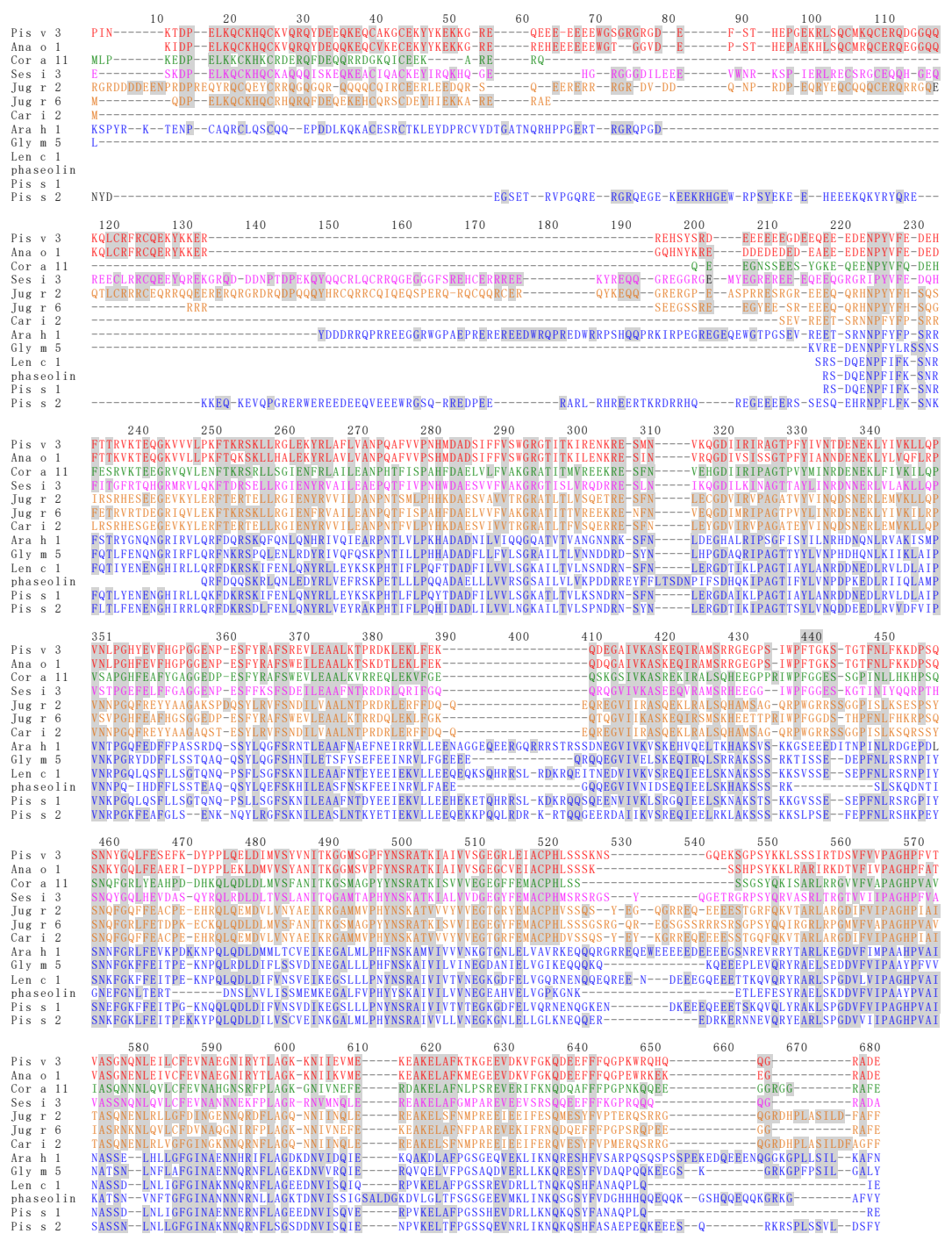

Figure 10. Multiple amino acid sequence alignment of 7S globulin/vicilin allergens Ana o 1 from Anacardium occidentale, Ara d 1 from Arachis duranensis, Car i 2 from Carya illinoinensis, Cic a 1 from 
Cicer arietinum, Coc $\mathrm{n} 1$ from Cocos nucifera, Cor a 11 from Corylus avellana, Gly m 5 and Gly m Bd 28K from Glycine max, Gos h Vic from Gossypium hirsutum, Jug n 2 from Juglans nigra, Jug r 2 and Jug r 6 from Juglans regia, Len c 1 from Lens culinaris, Lup a 1 from Lupinus albus, Lup an 1 from Lupinus angustifolius, phaseolin from Phaseolus vulgaris, Pin k 2 from Pinus koraiensis, Pis s 1 and Pis s 2 from Pisum sativum, Pis v 3 from Pistacia vera, Ses i 3 from Sesamum indicum, Zea m g1 from Zea mays. Identical amino acids are grey shadded. Aminoacid sequences of allergens belonging to the same botanical family, are similarly colored in red (Anacardiaceae), green (Betulaceae), magenta (Pedaliaceae), orange (Juglandaceae) and blue (Fabaceae).

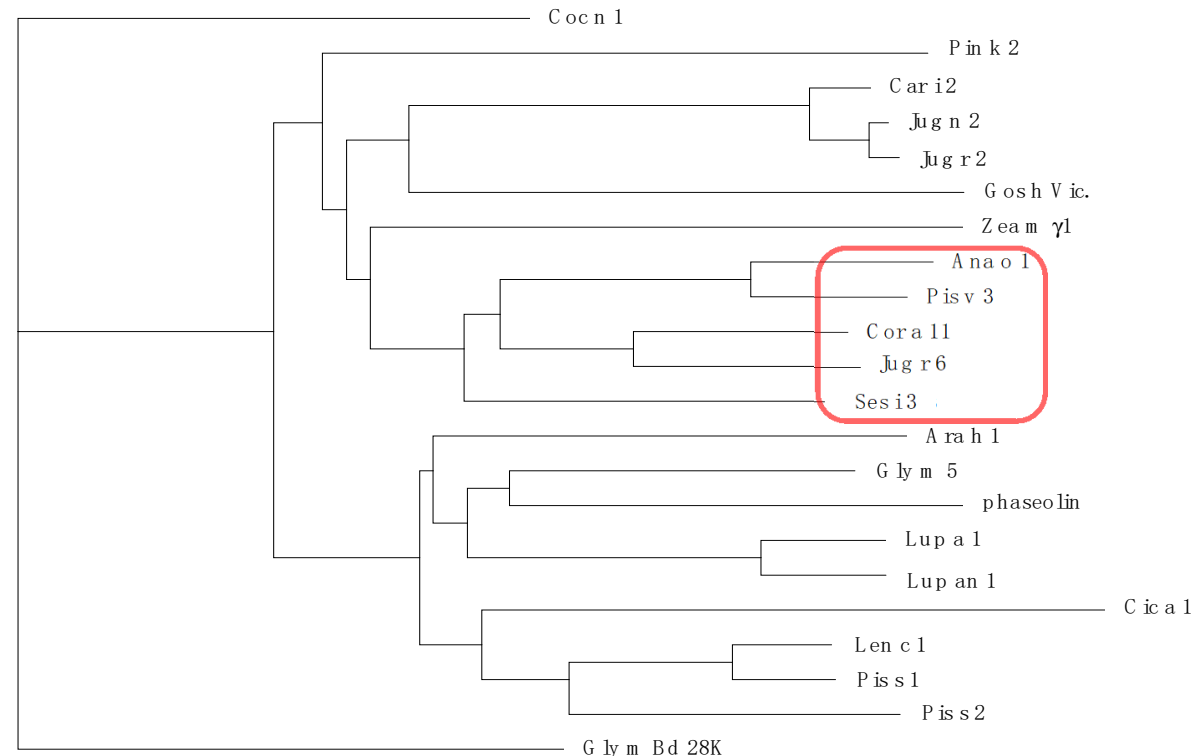

Figure 11. Dendrogram of 7S globulin/vicilin allergens from cashew nut (Ana o 1 from Anacardium occidentale), chickpea (Cic a 1 from Cicer arietinum), coconut (Coc $\mathrm{n} 1$ from Cocos nucifera), cotton (Gos $\mathrm{h}$ Vic. from Gossypium hirsutum), garden pea (Pis s 1 and Pis s 2 from Pisum sativum), hazelnut (Cor a 11 from Corylus avellana), kidney bean (phaseolin from Phaseolus vulgaris), Korean pine (Pin $\mathrm{k} 2$ from Pinus koraiensis), lentil (Len c 1 from Lens culinaris), lupine (Lup a 1 from Lupinus albus and Lup an 1 from L. annuus), maize (vicilin Zea $\mathrm{m} \gamma 1$ from Zea mays), peanut (Ara h 1 from Arachis hypogaea), pecan nut (Car i 2 from Carya illinoinensis), pistachio (Pis v 3 from Pistacia vera), pumpkin (Cuc ma 5 from Cucurbita maxima), sesamum (Ses i 3 from Sesamum indicum), soybean (Gly m 5 and Gly m Bd 28K from Glycine max), sunflower (Hel an 2S from Helianthus annuus), turnip and rapeseed (Bra $\mathrm{j} 1$ from Brassica juncea and Bra $\mathrm{r} 1$ from B. rapa; Bra $\mathrm{n} 1$ from B. napus), walnut (Jug n 2 from Juglans nigra, Jug r 2 and Jug r 6 from J. regia). Vicilins clustered to Pis v 1 are red circled.

The phylogenetic relationships observed between Pis v 3 and Ana o 1, are reflected in the similarity of their IgE-binding epitopic stretches along their amino acid sequences (Figure 12), that most probably accounts for the IgE-binding cross-reactivity reported between Pis v 3 and Ana o 1. Much less similarity occurs between Pis v 3 and other less phylogenetically closely related vicilin allergens like Jug r 2 (Juglandaceae), Ses i 3 (Pedaliaceae) and Ara h 1 (Fabaceae) (Figure 12). 


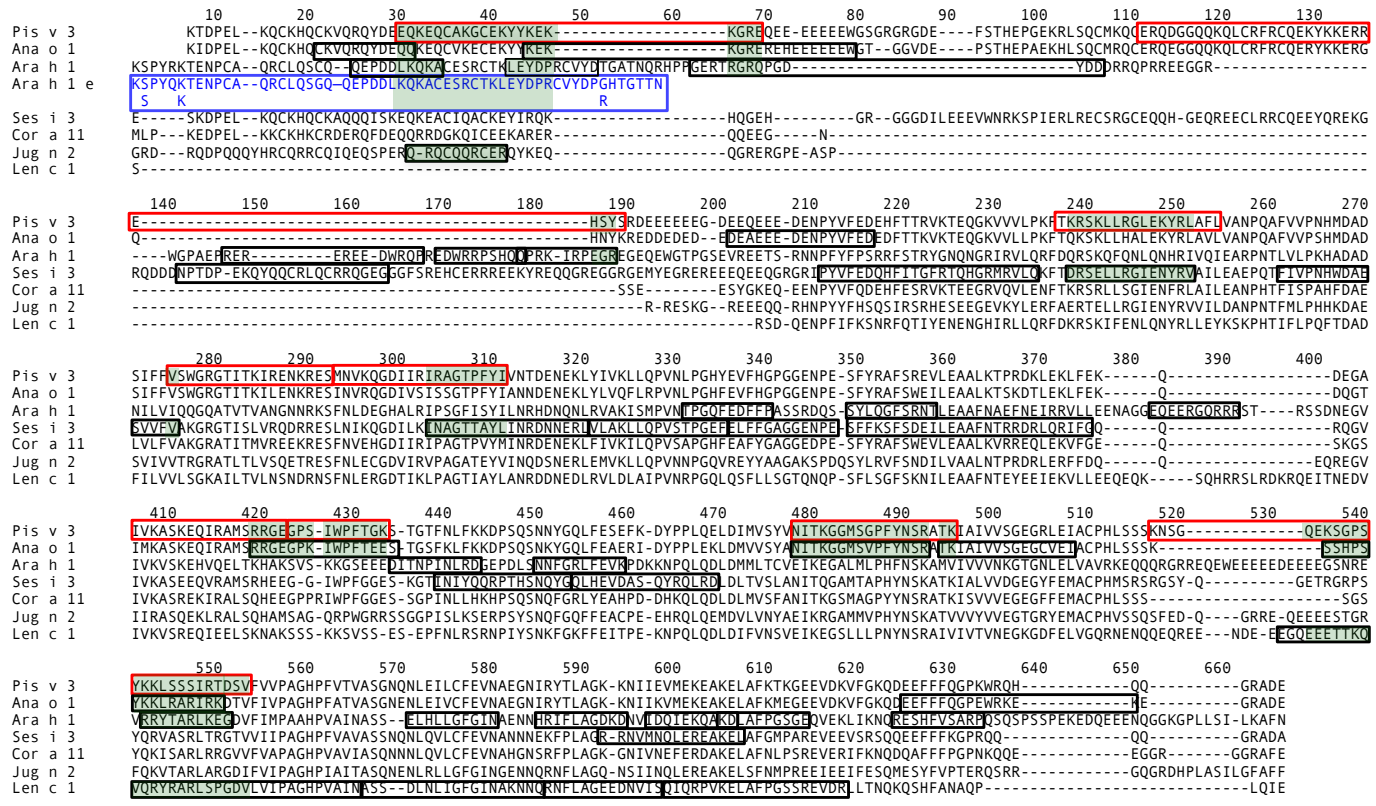

Figure 12. Multiple alignment of $7 \mathrm{~S}$ globulin/vicilin allergens Pis v 3, Ana o 1, Ara h 1, Ses i 3, Cor a 11, Jug n 2 and Len c 1 . Ara h 1 e, corresponding to the IgE-binding epitope recently identified at the $N$-terminal end of Ara h 1 sequence, contains 3 extra aminoacids compared to the $N$-terminal sequence of Ara $\mathrm{h}$ 1. The Ig-E-binding epitopes delineated along the amino acid sequence of Pis $\mathrm{v} 3$ (red boxes), Ara h 1 e (blue box), and Ana o 1, Ara h 1, Ses i 3, Cor a 11, Jug n 2 and Len c 1 (black boxes), are indicated. Amino acid residues occurring in regions of vicilins homologous to the IgE-binding epitopes of Pis v 3 , are shaded pale green.

Interestingly, the first epitopic stretch identified at the $\mathrm{N}$-terminal end of Pis v 3 amino acid sequence (epitope \#1), exhibits some amino acid identities with linear IgE-binding epitopes previously identified at the $N$-terminal end of Ana o 1, Ara h 1 and Len c 1 (Figure 12). This is also the case of an epitopic stretch recently characterized at the $\mathrm{N}$-terminus (26-83) of Ara $\mathrm{h} 1$, recognized as a major epitope responsible for the IgE-binding activity of the $7 \mathrm{~S}$ basic peanut protein fraction [58]. In fact, this IgE-binding epitope coincides with two other linear IgE-binding epitopes that had been previously identified along the Ara h 1 amino acid sequence $[59,60]$.

\subsection{Pis $v 2$ and Closely Related $11 S$ Globulins (Legumins)}

The cupin allergen Pis v 2 consists of a legumin homotrimer resulting from the non covalent association of three protomers built up from two cupin motifs (Figure 13C,F). In fact, each protomer consists of a large acidic $N$-terminal and a shorter basic $C$-terminal subunit covalently associated by a single disulfide bridge. Finally, two homotrimers associated face to face to build an hexameric structure corresponding to a dimer of homotrimers. Like in other legumin hexamers [57], the face to face association of both homotrimers should predominantly result from electrostatic interactions occurring between the oppositely charged faces of the homotrimers, one face being predominantly electronegative (colored blue) whereas the other is essentially electropositive (colored red) (Figure 13E,H).

Up to ten main IgE-binding epitopic stretches were identified along the amino acid sequence of the Pis v 2 protomer together with single epitopic spots (colored grey in Figure 13B), using the Spot method (Figure 13A,B). They correspond to IgE-binding patches well exposed at the surface of Pis $\mathrm{v}$ 2, except for epitopes \#1 (colored red) and \#3 (colored green), which are predominantly buried and much less exposed on the surface (Figure 13C,D,F,G). As with other allergens with cupin motifs, the more exposed IgE-binding patches coincide with the localization of electropositively (colored blue) and electronegatively (colored red) regions on the the molecular surface (Figure 13E,H). 
A

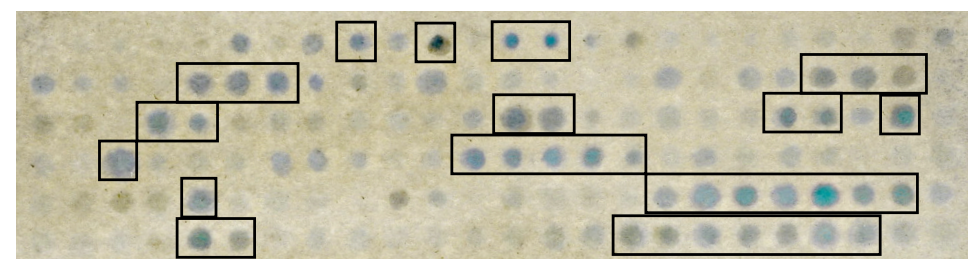

B

QIEQVVNSQQRQQQQRFQTQCQIQNLNALEPKRRIESEAGVTEFWDQNEEQLQ CANVAVFRHTIQSRGLLVPSYNNAPELVYVVOGSGIHGAVFPGCPETFQEESO SQSRSQHSRSERSQQSGEOHOKVRHIREGDIIALPAGVAHWIYNNGQSKLVLV ALADVGNSENQLDQYLRKFVLGGSPQQEIQGGGQSWSQSRSSRKGQQSNNILS AFDEEILAQSLNIDTQLVKKLQREEKQRGI IVRVKEDLQVLSPQRQEKEYSDN GLEETFCTMTLKLNINDPSRADVYNPRGGRVTSINALNLPILRFLQLSVEKGV LYQNAIMAP HWNMNAHSIVYITRGNGRMQIVSENGESVFDEEIREGQLVVVPQ NFAVVKRASSDGFEWVSFKTNGLAKISQLAGRISVMRGLPLDVIONSFDISRE DAWNLKESRSEMTIFAPGSRSQRQRN

C

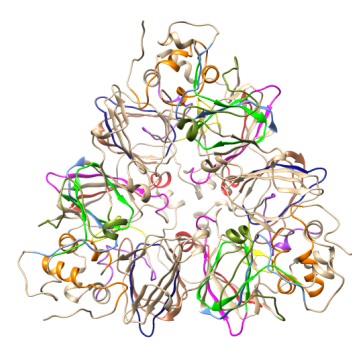

$\mathrm{F}$

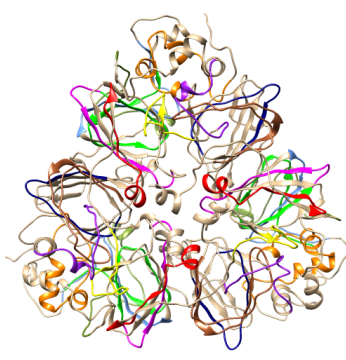

D

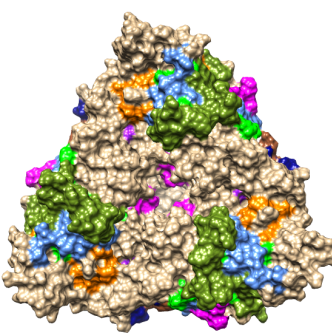

G
E

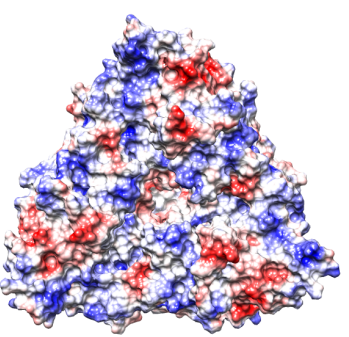

$\mathrm{H}$

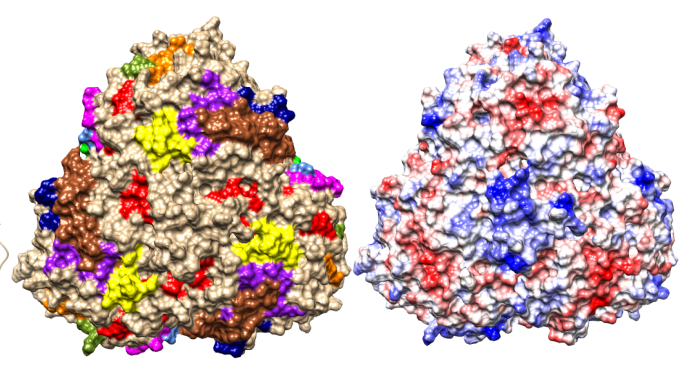

Figure 13. (A) IgE-binding peptides (boxed violet spots) revealed on the Pis $\mathrm{v} 2$ Spot membrane. (B) Mapping of the corresponding continuous IgE-binding epitopic regions (colored boxed white letters) along the amino acid sequence of Pis v 2. Epitopes are colored red (epitope \#1), blue (epitope \#2), green (epitope \#3), magenta (epitope \#4), yellow (epitope \#5), purple (epitope \#6), olive green (epitope \#7), sienna (epitope \#8), dark blue (epitope \#9) and orange (epitope \#10), respectively. (C,F) Ribbon diagram of the front (C) and back (F) face of the modelled Pis $v 2$ showing the localization of the colored IgE-binding epitopic regions. Epitopes are numbered and colored as in (B). (D,G) Surfaces occupied by the colored IgE-binding epitopes on the front (D) and back (G) face of Pis v 2. Epitopes are numbered and colored as in $(\mathbf{B}) .(\mathbf{E}, \mathbf{H})$ Mapping of Coulombic charges on the front $(\mathbf{E})$ and back (H) faces of Pis v 2. Electropositive and electronegative regions are colored blue and red, respectively. Neutral regions are white.

Ten distinct IgE-binding epitopic stretches were revealed along the amino acid sequence of Ses i 6 , the legumin allergen from sesame, associated to some IgE-binding single-spots, using the Spot method (Figure 14A,B). Except for epitope \#2 (colored blue), other epitopes \#1, \#3, \#4, \#5, \#6, \#7, \#8, \#9 and 
\#10, are nicely exposed on the surface of the Ses i 6 homotrimer (Figure 14C,D,F,G) and most of them coincide with electropositively (colored blue) and electronegatively (colored red) charged regions on the surface of Ses i 6 (Figure 14E,H).

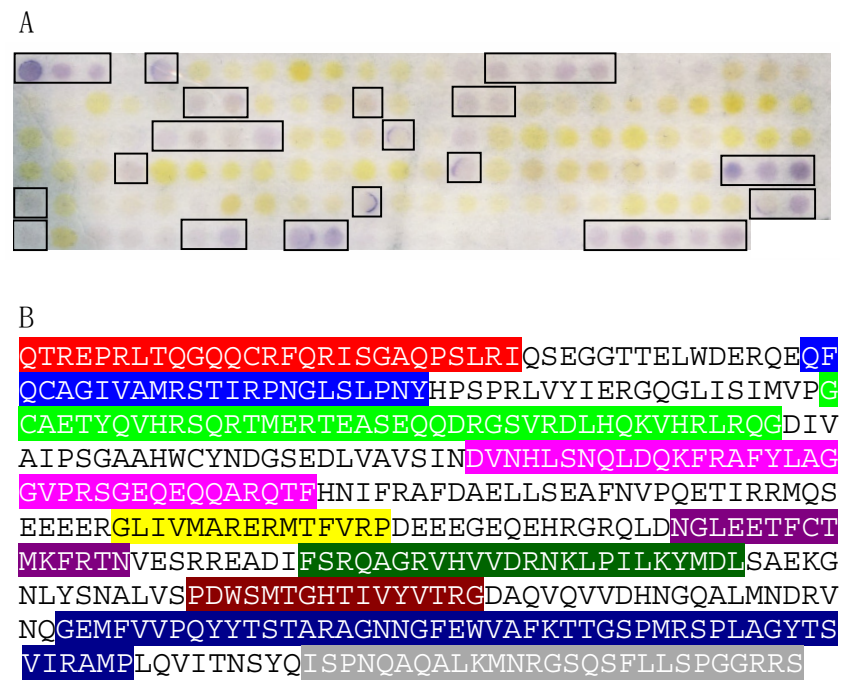

C

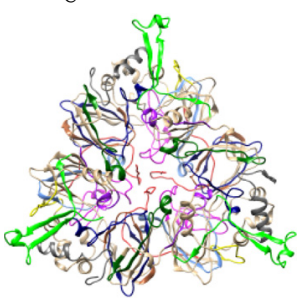

D

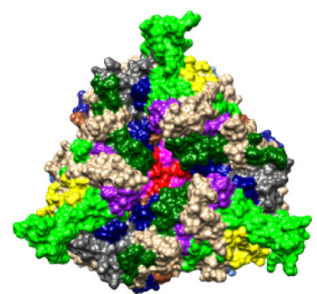

E

F

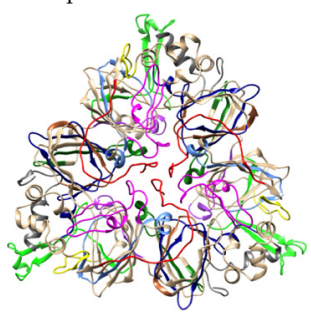

G

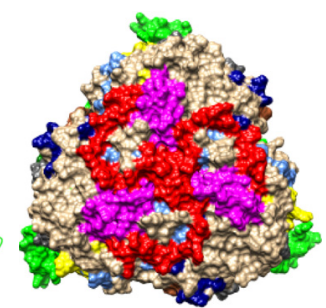

H

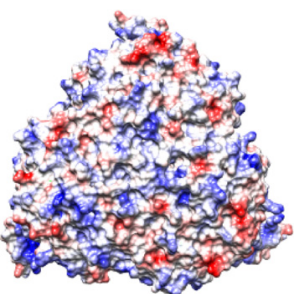

Figure 14. (A) IgE-binding peptides (boxed violet spots) revealed on the Ses i 6 Spot membrane. (B) Mapping of the corresponding continuous IgE-binding epitopic regions (colored boxed white letters) along the amino acid sequence of Ses i 6. Epitopes are colored red (epitope \#1), blue (epitope \#2), green (epitope \#3), magenta (epitope \#4), yellow (epitope \#5), purple (epitope \#6), deep green (epitope \#7), sienna (epitope \#8), dark blue (epitope \#9) and grey (epitope \#10), respectively. (C,F). Ribbon diagram of the front $(C)$ and back $(F)$ face of the modelled Ses i 6 showing the localization of the colored IgE-binding epitopic regions. Epitopes are numbered and colored as in (B). (D,G). Surfaces occupied by the colored IgE-binding epitopes on the front $(\mathbf{D})$ and back $(\mathrm{G})$ faces of Pis v 1. Epitopes are numbered and colored as in $(\mathbf{B})$. $(\mathbf{E}, \mathbf{H})$ Mapping of Coulombic charges on the front $(\mathbf{E})$ and back (H) faces of Ses i 6. Electropositive and electronegative regions are colored blue and red, respectively. Neutral regions are white.

Multiple amino acid sequence alignment of 115 globulin/legumin allergens shows a high degree of both identity and homology/similarity between the members of this protein family, especially between the members of the Anacardiaceae (Ana o 2, Pis v 2, Pis v 5), Juglandaceae (Jug n 4, Jug r 4) and Betulaceae (Cor a 9) families (Figure 15). Accordingly, both Ana o 2, Pis v 5, Jug n 4, Jug r 4 and Cor 
a 9, are closely clustered in the dendrogram built for the 11S globulin/legumin allergens (Figure 16). However, Pis v 2 appears as being more distantly related to Ana o 2, compared to Pis v 5.

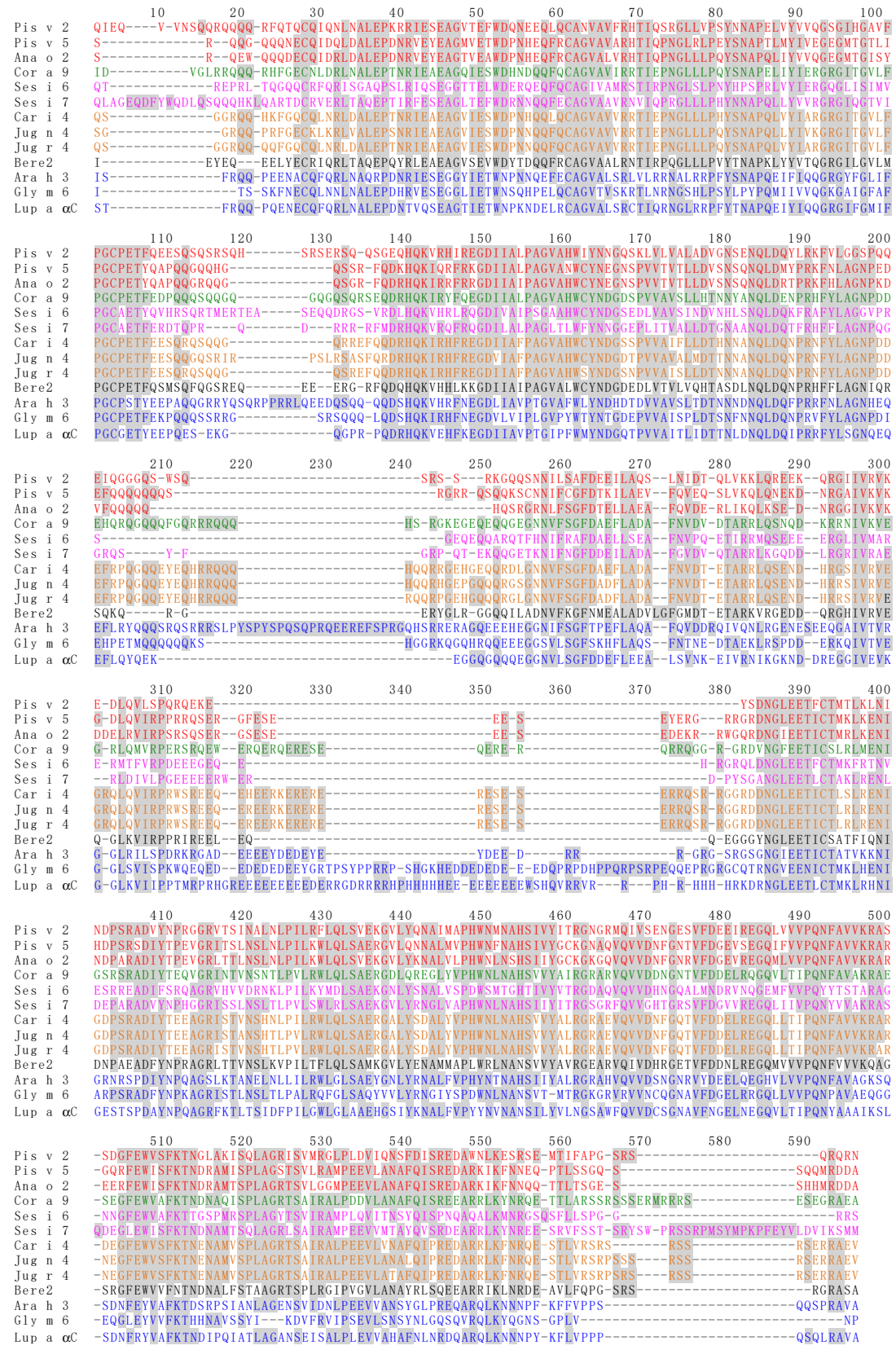

Figure 15. Multiple amino acid sequence alignment of $11 S$ globulin/legumin allergens Act c 12 from Actinidia chinensis, Ana o 2 from Anacardium occidentale, Ara h 3 from Arachis hypogaea, Ave s 11S from Avena sativa, Ber e 2 from Bertholletia excelsa, Car i 4 from Carya illinoinensis, Cic a 6 from Cicer arietinum, Coc $\mathrm{n} 4$ from Cocos nucifera, Cor a 9 from Corylus avellana, Cuc ma 4 from Cucurbita maxima, Fag t 1 from Fagopyrum tataricum, Gly m 6 from Glycine max, Jug n 4 from Juglans nigra, Jug r 4 from Juglans regia, Lup a $\alpha \mathrm{C}$ from Lupinus albus, Lup an $\alpha \mathrm{C}$ from Lupinus angustifolius, Pis v 2 and Pis v 5 from Pistacia vera, Pru du 6, Ses i 6 and Sesi i 7 from Sesamum indicum, Sin a 2 from Sinapis alba. Identical amino acids are grey shaded. Amino acid sequences of allergens belonging to the same botanical family, are similarly colored in red (Anacardiaceae), green (Betulaceae), magenta (Pedaliaceae), orange (Juglandaceae) and blue (Fabaceae). 


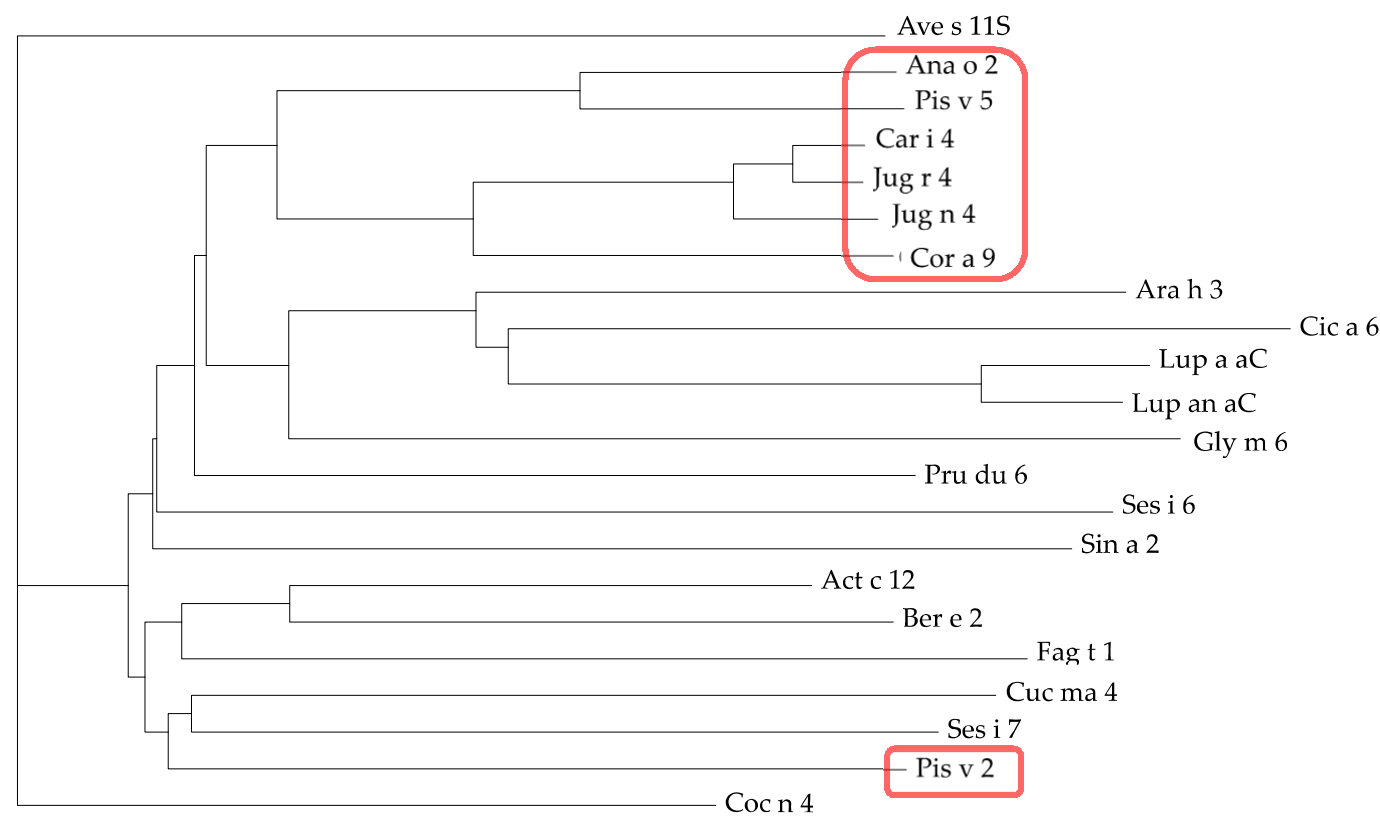

Figure 16. Dendrogram of $11 \mathrm{~S}$ globulin/legumin allergens from almond (Pru du 6 from Prunus dulcis), Brazil nut (Ber e 2 from Bertholletia excelsia), buckwheat (Fag t 1 from Fagopyrum tartaricum), cashew nut (Ana o 2 from Anacardium occidentale), chickpea (Cic a 6 from Cicer arietinum), coconut (Coc $\mathrm{n} 4$ from Cocos nucifera), hazelnut (Cor a 9 from Corylus avellana), kiwi (Act c 12 from Actinidia chinensis), lupine (convicilin Lup a $\alpha \mathrm{C}$ from Lupinus albus, convicilin Lup an $\alpha \mathrm{C}$ from Lupinus angustifolius, mustard (Sin a 2 from Sinapis alba), oat (Ave s 11S from Avena sativa), peanut (Ara h 3 from Arachis hypogaea), pecan nut (Car i 4 from Carya illinoinensis), pistachio (Pis v 2 and Pis v 5 from Pistacia vera), pumpkin (Cuc ma 4 from Cucurbita maxima), sesamum (Ses i 6 and Ses i 7 from Sesamum indicum), soybean (Gly $\mathrm{m} 6$ from Glycine max), walnut (Jug n 4 from Juglans nigra and Jug r 4 from J. regia). Legumins clustered to Pis v 1 are red circled.

In agreement with the phylogenetic relationships observed between Pis v 2, Pis v 5, Ana o 2, Jug $n$ 4, Jug $\mathrm{r} 4$, and Cor a 9, the sequential IgE-binding epitopic stretches identified along their amino acid sequences exhibit pronounced similarities (Figure 17), that most probably account for the IgE-binding cross-reactivity reported between some of these legumin allergens [61]. However, less similarity occurs between Pis v 2 and other phylogenetically related legumin allergens whereas Pis v 5 , the other legumin allergen from pistachio, appears as more closely related to other legumin allergens than Pis $\mathrm{v}$ 2. Compared to Pis v 2, Pis v 5 exhibits a very similar amino acid sequence with, however, a few amino acid changes. In spite of these changes, regions in Pis $v 5$ corresponding to the IgE-binding epitopes identified in Pis v 2, look like very similar (Figure 17). Conversely, Ara h 3, which belongs to the rather distantly related Fabaceae family, exhibits a higher degree of epitopic similarity with the other legumin allergens (Figure 17).

\subsection{Structural Similarities Observed among the Tree Nut Epitopic Regions}

Due to the high degree of conservation and phylogenetic relatedness of their amino acid sequences, $2 \mathrm{~S}$ albumin, vicilin and legumin allergens, are readily superposable with Rmsd values below $1.0 \AA$, e.g., for the pruned atom pairs corresponding to the conserved cupin motifs of the Pis v 3/Ana o 1 (0.906), Pis v 3/Jug r 2 (0.979), Pis v 3/Ses i 3 (0.955) and Pis v 3/Ara h 2 (0.929) vicilin pairs, respectively (Figure 18). 


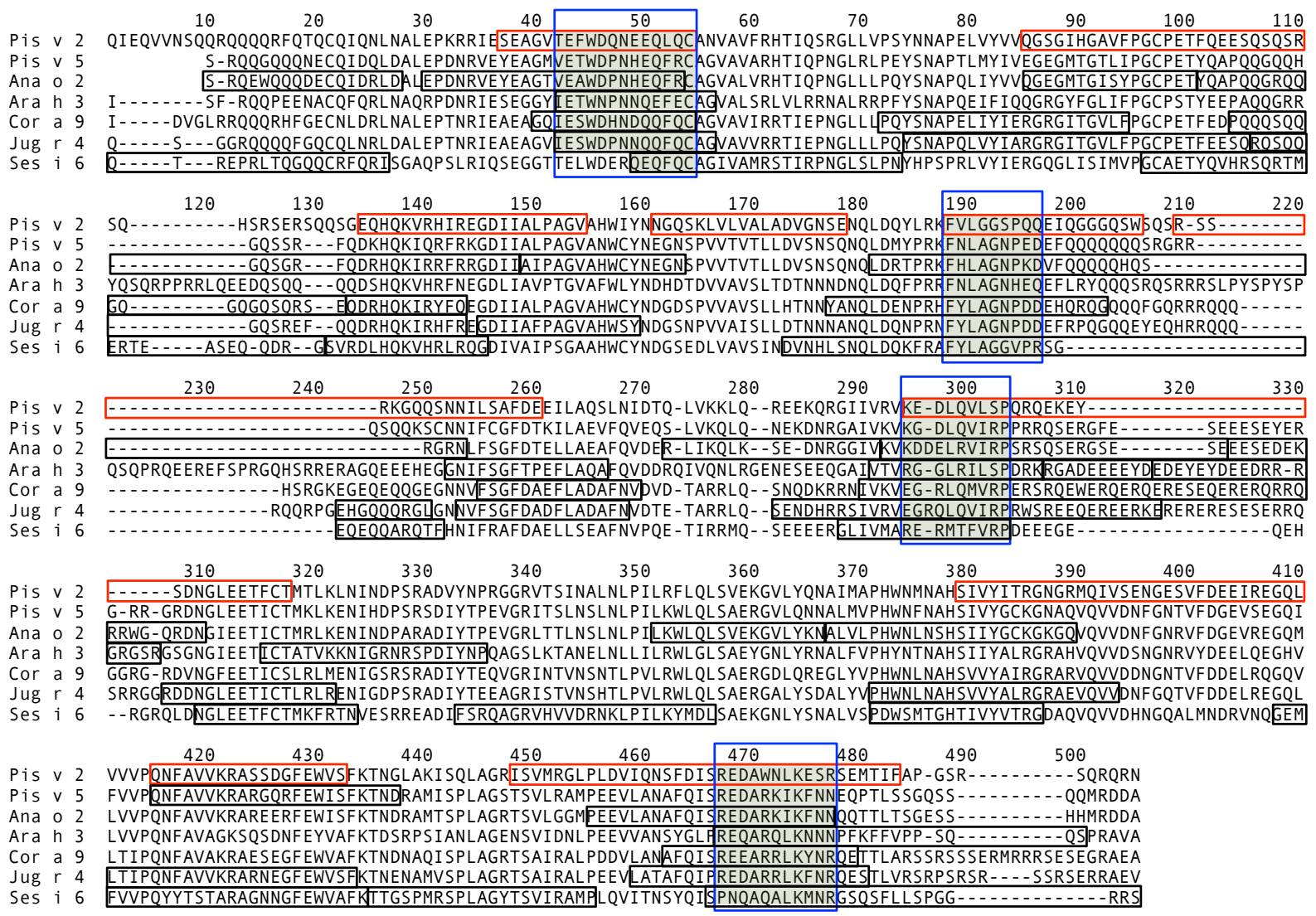

Figure 17. Multiple alignment of 11 S globulin/legumin allergens of Pis v 2, Pis v 5, Ana o 2, Ara h 3 , Cor a 9, Jug r 4 and Ses i 6 . The Ig-E-binding epitopes delineated along the amino acid sequence of Pis v 2 (red boxes) and Ana o 2, Ara h 3, Cor a 9, Jug r 4, Ses i 6 (black boxes) are indicated. Amino acid residues occurring in regions of legumins homologous to the IgE-binding epitopes of Pis v 2, are shaded green. Amino acid residues common to all the legumin sequences (blue boxes) are indicated.

A

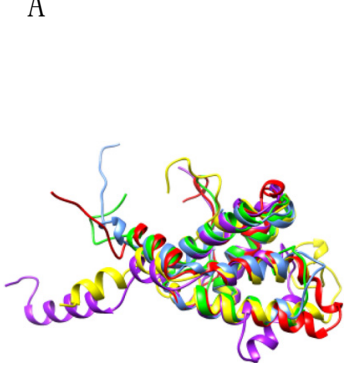

B

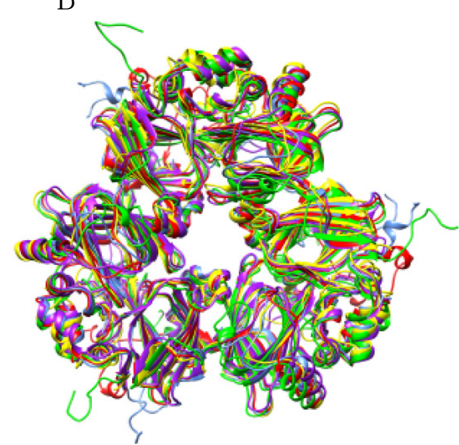

C

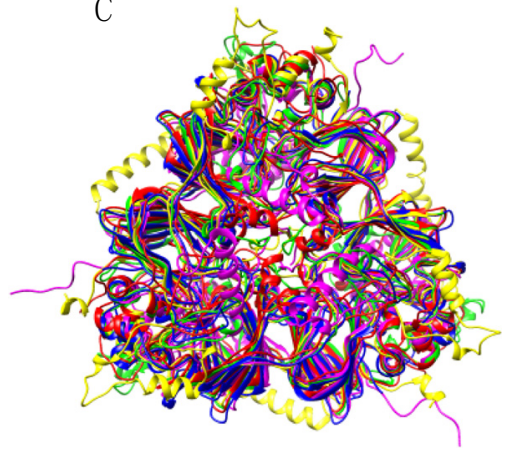

Figure 18. (A) Superposition of the ribbon diagrams of the $2 \mathrm{~S}$ albumin allergens Pis $v 1$ (colored red), Anao 3 (colored blue), Ara h 2 (colored yellow), Jug r 1 (colored green) and Ses i 1 (colored purple). (B) Superposition of the ribbon diagrams of the 7S globulin/vicilin allergens Pis v 3 (colored red), Ana o 1 (colored blue), Ara h 1 (colored yellow), Jug r 2 (colored green) and Ses i 3 (colored purple). (C) Superposition of the ribbon diagrams of the $11 \mathrm{~S}$ globulin/legumin allergens Pis v 2 (colored red), Ana o 2 (colored blue), Ara h 3 (colored yellow), Jug r 4 (colored green) and Ses i 6 (colored purple).

A detailed surface analysis of epitope \#1 of Pis $\mathrm{v} 1$ reveals that it shares a similar topographical distribution of the amino acid residues along the $\alpha$-helix, associated to partial conformational similarities, with the corresponding epitope \#1 from Ana o 3 (Figure 19A,B). Similarly, epitope \#8 from Pis v 2, 
exhibits a similar topographical distribution of amino acids and partial conformational similarities with the corresponding epitope from Ana o 2 (Figure 19C). Obviously, these epitopic similarities should account for the IgE-binding cross-reactivity often reported among the different allergens from pistachio and cashew nut [61]. However, these conformational similarities could only be observed between closely related epitopes sharing a high degree of identity. In other cases of couple of epitopes sharing a moderate level of amino acid sequence identity, no relevant conformational similarities could be identified.

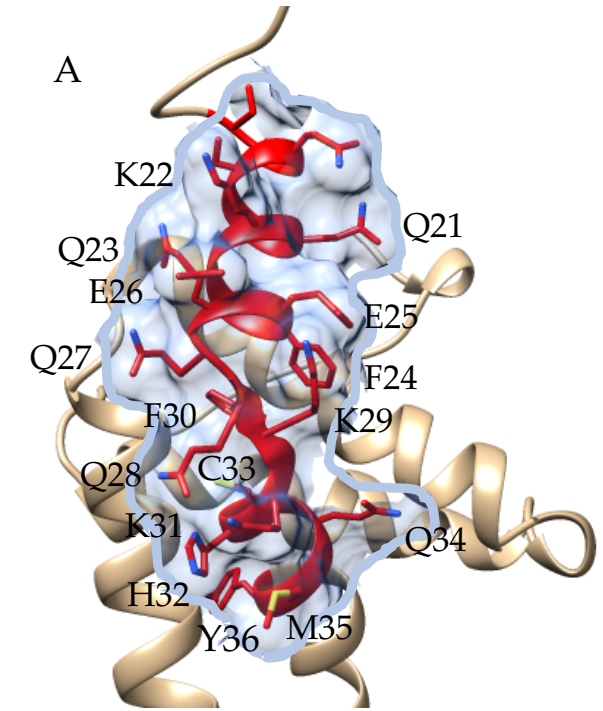

21QKQFEEQQKFKHCQMY36

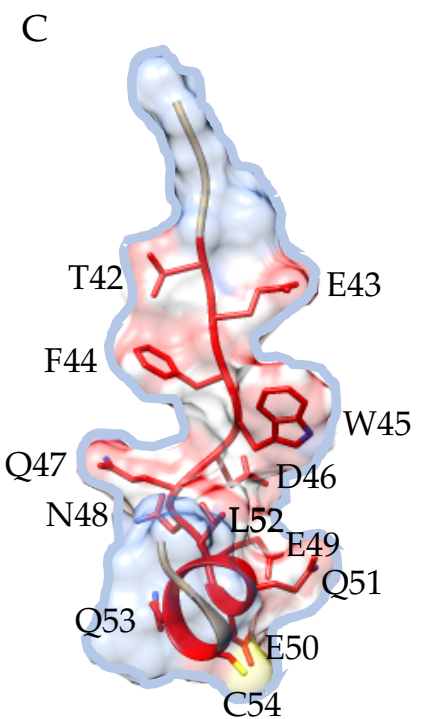

42TEFWDQNEEQLQC54

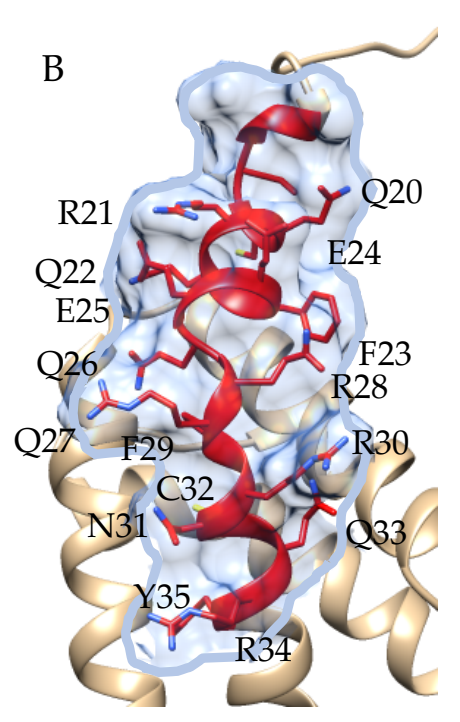

20QRQFEEQQRFRNCQRY35

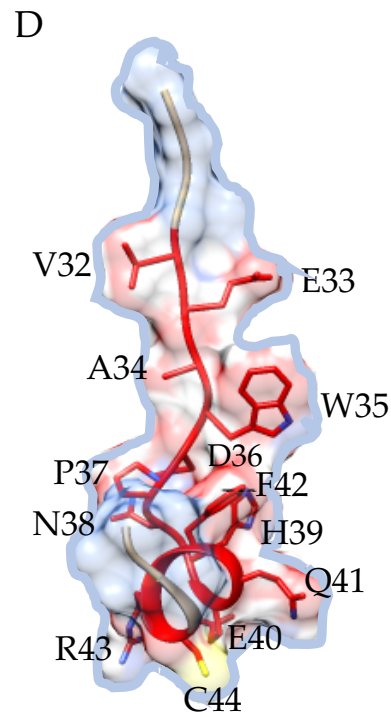

32VEAWDPNHEQFRC44

Figure 19. (A,B) Analysis of the topographical distribution and conformational similarities of amino acids of epitope \#1 from Pis v 1 (A), compared to epitope \#1 from Ana o 3 (B). The amino acid sequences of epitopes \#1 from Pis v 1 and Ana o 3, are indicated. (C,D) Analysis of the topographical distribution and conformational similarities of amino acids of epitope \#8 from Pis v 2 (C), compared to epitope \#8 from Ana o 2 (D). The amino acid sequences of epitopes \#8 from Pis v 2 and Ana o 2, are indicated. 
In addition to the IgE-binding epitopic similarities observed among the different members of the same allergen family, other cross-reactivity has been previously reported between the $\mathrm{CD}_{4}(+) \mathrm{T}$ cell epitopes from cashew nut, hazelnut and pistachio [62].

These IgE-binding epitopic amino acid identities, sometimes associated with conformational similarities, come in support to the well recognized cross-reactivities among the allergens from different species belonging to the same family of Anacardiaceae (pistachio, cashew), Juglandaceae (walnut), Fabaceae (peanut), Pedaliaceae (sesamum) and Betulaceae (hazelnut), previously reported from immunodiffusion and crossed immuno-electrophoresis experiments, Western blotting experiments, recognition of recombinant allergens and clinical assessments [63-65] (Figure 20).

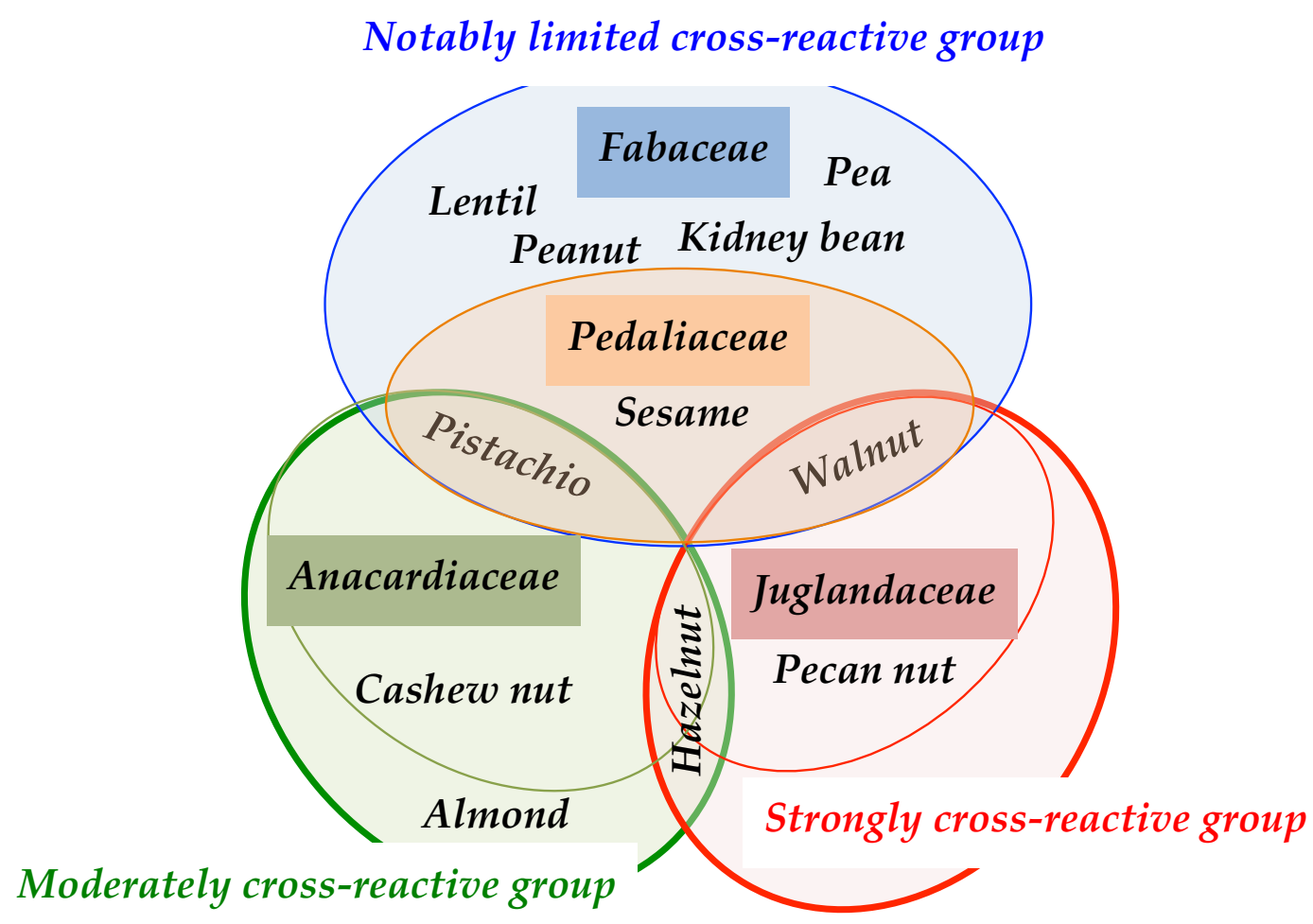

Figure 20. Cartoon summarizing the different types, limited, mild or strong, of cross-reactivities susceptible to occur between the members of the different families of Anacardiaceae (green), Juglandaceae (red), Fabaceae (blue) and Pedaliaceae (orange). Hazelnut belongs to the Betulaceae.

\subsection{Predicted Resistance of IgE-Binding Epitopic Regions to Proteolysis}

The allergenicity of the vicilin allergen Ara $\mathrm{h} 1$ from peanut (Arachis hypogaea), has been attributed in part to the particular arrangement of the monomers in the typical homotrimeric structure of vicilin/7S globulin proteins [66]. The quaternary association of monomers in the homotrimer has been proved to mainly depends on hydrophobic interactions responsible for the swapping of monomers by their distal ends, allowing the major IgE-binding epitopes located along these buried extremities to escape the proteolytic degradation by digestive enzymes. However, in the case of Pis v 3 (vicilin) and Pis v 2 (legumin), an additional mechanism could explain the resistance to digestive proteolysis. Looking at the distribution of the putative cleavage sites for pepsin predicted to occur at the molecular surface of Pis v 3 and Pis v 2, using the web server PeptideCutter of Expasy (https://web.expasy.org/peptide_cutter/, accessed on 15 January 2021), suggests that some of the IgE-binding epitopes previously identified does not contain cleavage sites and could remain in attacked by the protease, e.g., epitopes \#3, \#4, \#6, \#9 from Pis v 3, and epitopes \#1,\#2, \#3, \#5, \#6, \#7 from Pis v 2 (Figure 21). 
A

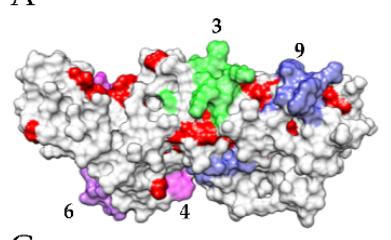

C

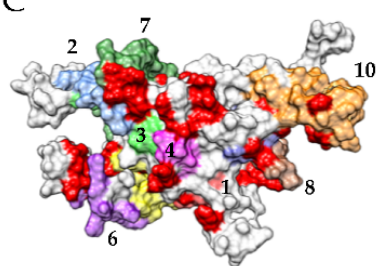

B

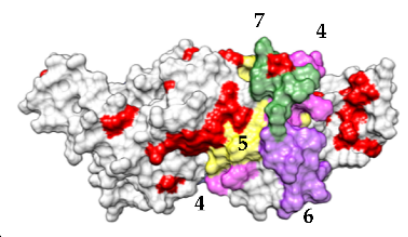

D

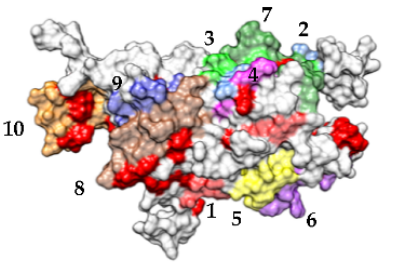

Figure 21. (A-D). Localization of predicted cleavage sites by pepsin (red patches) on the three-dimensional surface of front (A) and back face (B) of Pis v 3 , and front (C) and back face (D) of Pis v 2 monomers. The IgE-binding epitopic regions identified on the surface of Pis v 3 are numbered and colored pale green (epitope \#3), magenta (epitope \#4), yellow (epitope \#5), purple (epitope \#6), dark green (epitope \#7), brown (epitope \#8) and dark blue (epitope \#9), respectively. The IgE-binding epitopic regions identified on the surface of Pis $\mathrm{v} 2$ are numbered and colored red (epitope $\# 1$ ), pale blue (epitope \#2), pale green (epitope \#3), magenta (epitope \#4), yellow (epitope \#5), purple (epitope \#6), dark green (epitope \#7), brown (epitope \#8), dark blue (epitope \#9) and orange (epitope $\# 10)$, respectively.

However, due to the occurrence of numerous putative cleavage sites for trypsin predicted in the IgE-binding epitopes of both Pis v 3 and Pis v 2, most of these epitopes should be degraded later, during the intestinal digestion in the presence of trypsin, except for epitopes \#1, \#2 and \#8 from Pis v 2, which do not contain any K or R residues (Figure 13).

\section{Discussion}

Using the Spot technique with a panel of IgE-containing sera from patients allergic to pistachio and cashew nut, sequential IgE-binding epitopes were identified on the molecular surface of the modelled Pis v 1 (2S albumin), Pis v 2 (legumin) and Pis v 3 (vicilin) allergens, respectively. These epitopic amino acid stretches essentially consist of hydrophilic ( $N, Q, S, T)$ and charged residues $(\mathrm{D}, \mathrm{E}, \mathrm{K}, \mathrm{R})$. In this respect, these residues account for $\sim 60 \%$ of the epitopic stretches identified on Pis v 3 . Accordingly, most of these epitopic regions coincide with the electropositively and electronegatively charged areas occurring on the surface of the allergens. Moreover, and whatever the size of the allergens, a few IgE-binding epitopes locate in the same area and their coalescence should create more extended epitopic surfaces susceptible to correspond to discontinuous epitopes. However, this clustering tendency is more or less pronounced depending on the allergens (see Figure 3D,G for Pis v 1, Figure 8D,G for Pis v 3, and Figure 13D,G for Pis v 2). A similar epitopic coalescence was observed for the counterpart allergens of Pis v 1 (Figure 4D,G for Ana o 3), Pis v 3 (Figure 9D,G for Ses I 3 ) and Pis v 2 (Figure 14D,G for Ses I 6). Finally, some IgE-binding epitopes well exposed on the surface of the cupin allergen protomers, e.g., epitope \#8 of Pis v 3 (which contains the putative N-glycosylation site at 243NIT), become partly buried upon the oligomeric association of the protomers. Accordingly, the allergenic potency of the Pis v 3 protomer should slightly differ from that of the Pis v 3 homotrimer. Obviously, these discrepancies depend on the mode of association of the protomers in the vicilin and legumin homotrimers [62]. In addition, the occurrence of a $\mathrm{N}$-glycan chain on each Pis v 3 protomer at the $\mathrm{N}$-glycosylation site 243NIT located on epitope \#8, which is predicted to be actually glycosylated by GlyProt (http://www.glycosciences.de/modeling/glyprot/php/main.php, accessed on 15 January 2021) server, should participate in the allergenic potency of the allergen. $\mathrm{N}$-glycans have been demonstrated to participate in the IgE-binding activity of $\mathrm{N}$-glycosylated epitopes of e.g., the structurally related Ara $\mathrm{h} 1$ vicilin allergen from peanut [63]. The $N$-glycan chain of the 13NLS glycosylation site of Pis v 1 , 
which is adjacent to the IgE-binding epitope \#1, could similarly participate in the allergenicity as a carbohydrate determinant (CDD), even though there are no evidence for such a role of the $N$-glycan chains of pistachio allergens.

The approach which has been used to identify the linear or continuous epitopes, which combines the Spot technique with molecular modelling, provides results which need to be interpreted with caution since it does not allow to identify discontinuous or conformational epitopes, depending on the length of the synthetic peptides used as IgE-binding probes. In addition, while the molecular modelling techniques have improved significantly during the past decade, they still remain less reliable than the conventional approaches by X-ray crystallography or NMR to identify and map the IgE-binding epitopes on the molecular surface of the allergen-IgE complexes. However, the coupling of Spot and molecular modelling techniques is a reasonable and expedient compromise to get an insight into the IgE-binding epitopes from allergenic proteins.

Depending on the localization of the exposed pepsin and trypsin cleavage sites on the three-dimensional model built for the Pis $\mathrm{v} 3$ and Pis v 2 protomers (Figure 21), a central core structure remaining unaltered upon the digestive proteolytic attack by pepsin is predicted to occur in both allergens. Interestingly, this core structure still retains some epitopes that should remain protected from the proteolytic attack till Pis $\mathrm{v} 3$ is recognized by the immuno-competent cells of the gastro-intestinal tract. However, except for a few IgE-binding epitopic stretches from Pis v 2, apparently devoid of lysine and arginine residues, most of the epitopes remaining unaltered upon the pepsin attack, should be further degraded due to their richness in electropositively charged residues.

A strong overall IgE-binding cross-reactivity between pistachio and cashew allergens has been often reported from immunoelectrophoretic analyses, Western blotting experiments, and specific IgE measurements [63-65]. Both vicilins (Pis v 3, Ana o 1) [11] and 2 S albumins (Pis v 1, Ana o 3) [67] have been incriminated as the main IgE-binding cross-reacting allergens. A detailed surface analysis of the epitopes occurring on the molecular surface of these allergens, revealed a rather high degree of amino acid sequence identities, associated or not with conformational similarities depending on the degree of sequence identity occurring between their epitopes. Both sequence identities and conformational similarities establish the molecular basis of the IgE-binding cross-reactivity between the allergens from pistachio and cashew, which belong to the same botanical family of Anacardiaceae. However, the surface analysis performed on the legumin allergens revealed no conformational similarities between most of the sequential IgE-binding epitopes of Pis v 2 and Ana o 2. A conformational IgE-binding epitope (called 2B5) was identified on the molecular surface of Ana o 2 that mainly consists of 24 amino acid residues (20EPDNRVEYEAGTVEAWDPNHEQFR43) located at the $N$-terminus of the large (acidic) subunit, which is expressed only when associated to the small (basic) subunit $[68,69]$. The IgE-binding epitopes \#1 and \#2 of Pis $v 2$ were identified on the corresponding amino acid sequence stretch (30EPKRRIESEAGVTEFWDQNEEQLQ53) which displays 55\% identity (underlined bold letters) with the $2 \mathrm{~B} 5$ epitope.

Obviously, the amino acid sequence identities between the IgE-binding epitopes from allergens of different origin depend on the degree of conservation the allergens have retained during evolution. Accordingly, the occurrence of IgE-binding epitopes sharing similar physicochemical properties has been pointed out as a key factor contributing to the cross-reactivity among peanut and tree nut allergens [61]. In this respect, $2 \mathrm{~S}$ albumin allergens (Pis v 1, Ana o 3) and vicilin allergens (Pis v 3, Ana o 1), are predicted to display a degree of conservation higher than that predicted for the legumin allergens (Pis v 2, Ana o 2). Most conserved residues essentially occur along the structurally conserved secondary features, e.g., the $\alpha$-helices of $2 S$ albumins and the $\beta$-strand cupin motifs of the vicilins. These conserved regions are predominantly built up from hydrophilic residues. According to the sequence and conformational similarities observed among $2 \mathrm{~S}$ albumin and vicilin allergens, Pis $\mathrm{v} 1$ and Pis $\mathrm{v} 3$ of pistachio closely cluster with the corresponding Ana o 3 and Ana o 1 allergens of cashew in the dendrograms built up from the amino acid sequence alignments with the neighbor joining method (Figures 6 and 11). Although Pis v 2 and Ana o 2 allergens appear as being more distantly related 
in relation with the lower degree of sequence similarities that relate both allergens, another legumin allergen related to Pis v 2, Pis v 5, is closely clustered to Ana o 2 and other tree nut allergens in the legumin dendrogram (Figure 16).

Due to the high degree of conservation of the $2 \mathrm{~S}$ albumin and cupin allergen structures, the occurrence of amino acid sequence similarities observed between the pistachio allergens Pis v 1 , Pis v 2 and Pis $\mathrm{v} 3$ and other homologous allergens from either closely or distantly related families, has an influence on their allergenicity and cross-reactivity. Especially, the location of the amino acid sequence similarities in regions corresponding to IgE-binding epitopes, greatly determine their capacity to cross-react with the corresponding regions from other homologous allergens. Depending on the degree of sequence identity between epitopes belonging to homologous allergens, the resulting cross-reactivity will be strong, moderate or low. In this respect, the sequence similarities observed between the homologous allergens of Anacardiaceae, e.g., between Pis v 1 and Ana o 3 or between Pis v 2 and Ana o 2, account for the high level of cross-reactivity and cross-allergenicity observed between pistachio and cashew. The IgE-binding cross-reactivity the major allergens from pistachio, Pis $v 1$, Pis $v 2$ and Pis v 3, share with homologous proteins from peanut, tree nuts and proteins from, sesame, buckwheat, peppercorn or mango, has a clinical incidence and in particular, helps to discriminate between allergies and co-sensitizations $[19,22,70-78]$. Whereas co-sensitization frequently occurs between pistachio and cashew, it is clinically relevant in only one-third of cases [72]. However, skin prick tests (SPT) performed on French children indicated that a low reaction dose to cashew in cashew-allergic children would be a predictive factor of allergy to pistachio [78]. Accordingly, oral food challenge to pistachio should be avoided in cashew-allergic children exhibiting a low reaction dose to cashew nut.

Author Contributions: Methodology and validation, A.B., H.B.; investigation, C.N. and C.G.; software, P.R.; original-draft preparation, C.G. and P.R.; writing-review and editing, P.R. All authors have read and agreed to the published version of the manuscript.

Funding: This research received no external funding.

Informed Consent Statement: Informed consent was obtained from parents of subjects involved in the study.

Acknowledgments: This article is dedicated to the memory of Fabienne Rancé, who actively contributed to the methodology and validation of the results and provided us with the patient sera used in this work. Many thanks to Alain Jauneau (CNRS, Toulouse, France), who provided us with the micrography and histo-immunochemical pistachio slices.

Conflicts of Interest: The authors declare no conflict of interest.

\section{Abbreviations}

$\begin{array}{ll}\text { Act c } 12 & \text { Actinidia chinensis (kiwi) 11S globulin/legumin allergen } \\ \text { Ana o 1 } & \text { Anacardium occidentale (cashew) 7S globulin/vicilin allergen } \\ \text { Ana o 2 } & \text { Anacardium occidentale (cashew) 11S globulin/legumin allergen } \\ \text { Ana o 3 } & \text { Anacardium occidentale (cashew) 2S albumin allergen } \\ \text { Ara d 2/6 } & \text { Arachis duranensis (peanut) 2S albumin allergens } \\ \text { Ara h 1 } & \text { Arachis hypogaea (peanut) 7S globulin/vicilin allergen } \\ \text { Ara h 2/6/7 } & \text { Arachis hypogaea (peanut) 2S albumin allergens } \\ \text { Ara h 3 } & \text { Arachis hypogaea (peanut) 11S globulin/legumin allergen } \\ \text { Ara i 2/6 } & \text { Arachis ipaensis (peanut) allergens } \\ \text { Ave s 11S } & \text { Avena sativa (oat) 11S globulin/legumin allergen } \\ \text { Ber e 1 } & \text { Bertholletia excelsa (Brazil nut) 2S albumin allergen } \\ \text { Ber e 2 } & \text { Bertholletia excels (Brazil nut) 11S globulin/legumin allergen } \\ \text { Bra j 1 } & \text { Brassica juncea (brown mustard) 2S albumin allergen } \\ \text { Bra n 1 } & \text { Brassica napus (rapeseed) 2S albumin allergen } \\ \text { Bra r 1 } & \text { Brassica rapa (turnip) 2S albumin allergen } \\ \text { Car i 1 } & \text { Carya illinoinensis (Pecan nut) 2S albumin allergen } \\ \text { Car i 2 } & \text { Carya illinoinensis (Pecan nut) 7S globulin/vicilin allergen } \\ \text { Car i 4 } & \text { Carya illinoinensis (Pecan nut) 11S globulin/legumin allergen } \\ \text { Cic a 1 } & \text { Cicer arietinum (chickpea) 2S albumin allergen } \\ \text { Cic a 6 } & \text { Cicer arietinum (chickpea) 11S globulin/legumin allergen } \\ \text { Coc c 1 } & \text { Cocos nucifera (coconut) 7S globulin/vicilin allergen } \\ \text { Coc n } 4 & \text { Cocos nucifera (coconut) 11S globulin/legumin allergen } \\ \text { Cor a 14 } & \text { Corylys avellana (hazelnut) 2S albumin allergen } \\ \text { Cor a 11 } & \text { Corylus avellana (hazelnut) 7S globulin/vicilin allergen } \\ & \end{array}$




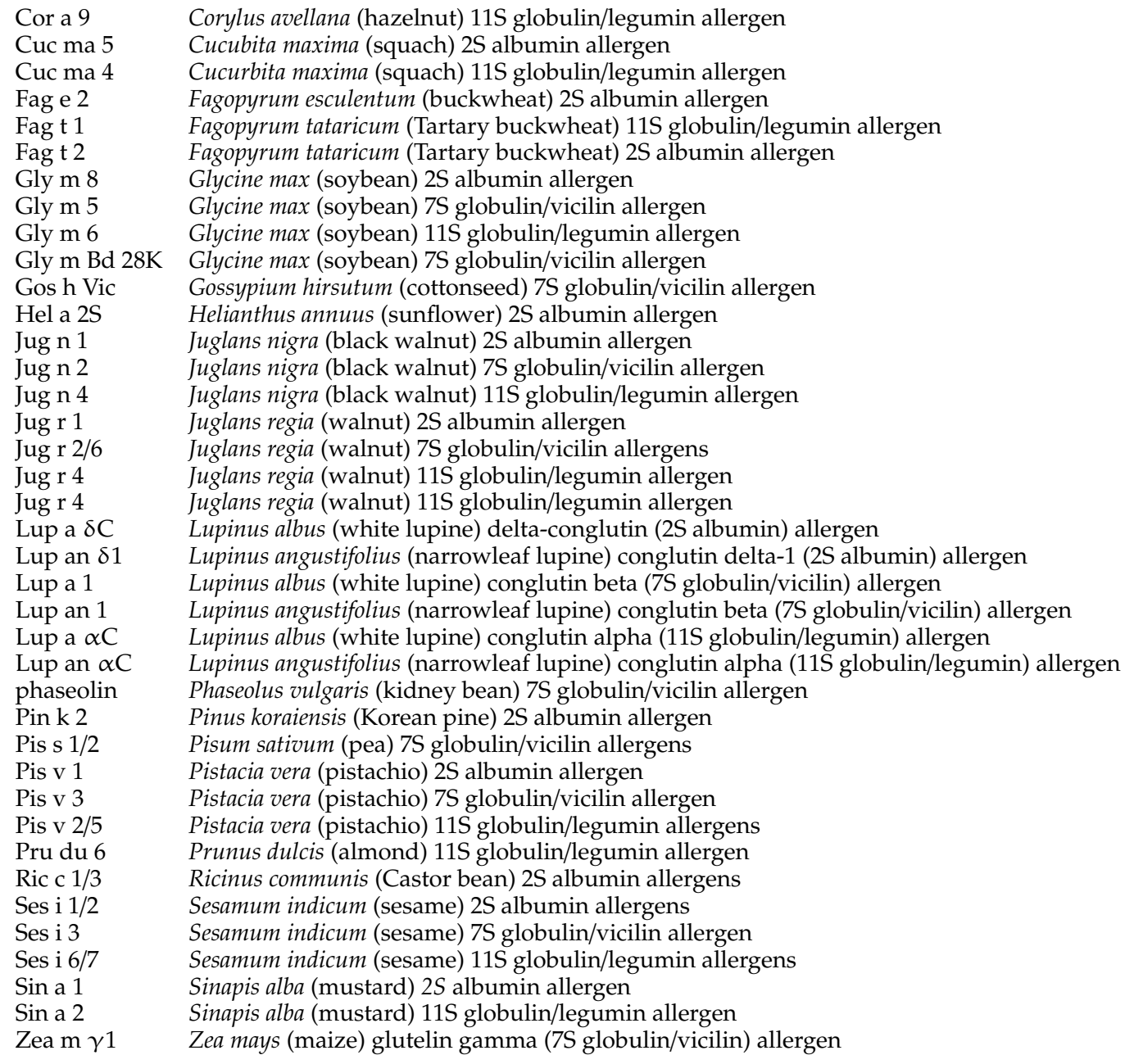

\section{References}

1. Husain, Z.; Schwartz, R.A. Food allergy update: More than a peanut of a problem. Int. J. Dermatol. 2013, 52, 386-394. [CrossRef]

2. Smeekens, J.M.; Bagley, K.; Kulis, M. Tree nut allergies: Allergen homology, cross-reactivity, and implications for therapy. Clin. Exp. Allergy 2018, 48, 762-772. [CrossRef]

3. Costa, J.; Silva, I.; Vicente, A.A.; Oliveira, M.B.P.P.; Mafra, I. Pistachio nut allergy: An updated overview. Crit. Food. Sci. Nutr. 2019, 59, 546-562. [CrossRef] [PubMed]

4. Geiselhart, S.; Hoffmann-Sommergruber, K.; Bublin, M. Tree nut allergens. Mol. Immunol. 2018, 100, 71-81. [CrossRef] [PubMed]

5. Teuber, S.S.; Beyer, K. Peanut, tree nut and seed allergies. Curr. Opin. Allergy Clin. Immunol. 2004, 4, $201-203$. [CrossRef]

6. Sicherer, S.H.; Muñoz-Furlong, A.; Godbold, J.H.; Sampson, H.A. US prevalence of self-reported peanut, tree nut, and sesame allergy: 11-year follow-up. J. Allergy Clin. Immunol. 2010, 125, 1322-1326. [CrossRef] [PubMed]

7. Porcel, S.; Sánchez, A.B.; Rodríguez, E.; Fletes, C.; Alvarado, M.; Jiménez, S.; Hernández, J. Food-dependent exercise-induced anaphylaxis to pistachio. J. Investig. Allergol. Clin. Immunol. 2006, 16, 71-73.

8. Rancé, F.; Bidat, E.; Bourrier, T.; Sabouraud, D. Cashew allergy: Observations of 42 children without associated peanut allergy. Allergy 2003, 58, 1311-1314. [CrossRef]

9. Fernandez, C.; Fiandor, A.; Martinez-Garate, A.; Martinez-Quesada, J. Allergy to pistachio: Cross-reactivity between pistachio nut and other Anacardiaceae. Clin. Exp. Allergy 1995, 25, 1254-1259. [CrossRef] 
10. Ahn, K.; Bardina, L.; Grishina, G.; Beyer, K.; Sampson, H.A. Identification of two pistachio allergens, Pis v 1 and Pis v 2, belonging to the $2 S$ albumin and 11 S globulin family. Clin. Exp. Allergy 2009, 39, 926-934. [CrossRef]

11. Willison, L.N.; Tawde, P.; Robotham, J.M.; Penney, R.M.; Teuber, S.S.; Sathe, S.K.; Roux, K.H. Pistachio vicilin, Pis v 3, is immunoglobulin E-reactive and cross-reacts with the homologous cashew allergen, Ana o 1. Clin. Exp. Allergy 2008, 38, 1229-1238. [CrossRef]

12. Ayuso, R.; Grishina, G.; Ahn, K.; Bardina, L.; Beyer, K.; Sampson, H.A. Identification of a MnSOD-like protein as a new major pistachio allergen. J. Allergy Clin. Immunol. 2007, 119, 115. [CrossRef]

13. Burks, A.W.; Cockrell, G.; Stanley, J.S.; Helm, R.M.; Bannon, G.A. Recombinant peanut allergen Ara h I expression and IgE binding in patients with peanut hypersensitivity. J. Clin. Investig. 1995, 96, 1715-1721. [CrossRef] [PubMed]

14. Viquez, O.M.; Summer, C.G.; Dodo, H.W. Isolation and molecular characterization of the first genomic clone of the major peanut allergen, Ara h 2. J. Allergy Clin. Immunol. 2001, 107, 713-717. [CrossRef] [PubMed]

15. Rabjohn, P.; Helm, E.M.; Stanley, J.S.; West, C.M.; Sampson, H.A.; Burks, A.W.; Bannon, G.A. Molecular cloning and epitope analysis of the peanut allergen Ara h 3. J. Clin. Investig. 1999, 103, 535-542. [CrossRef] [PubMed]

16. Bastiaan-Net, S.; Reitsma, M.; Cordewener, J.H.G.; van der Valk, J.P.M.; America, T.A.H.P.; Dubois, A.E.J.; Gerth van Wijk, R.; Savelkoul, H.F.J.; de Jong, N.W.; Wichers, H.J. IgE cross-reactivity of cashew nut allergens. Int. Arch. Allergy Immunol. 2019, 178, 19-32. [CrossRef] [PubMed]

17. Dreskin, S.C.; Koppelman, S.J.; Andorf, S.; Nadeau, K.C.; Kalra, A.; Braun, W.; Negi, S.S.; Chen, X.; Schein, C.H. The importance of the $2 \mathrm{~S}$ albumins for allergenicity and cross-reactivity of peanuts, tree nuts, and sesame seeds. J. Allergy Clin. Immunol. 2020. [CrossRef]

18. Sirvent, S.; Akotenou, M.; Cuesta-Herranz, J.; Vereda, A.; Rodríguez, R.; Villalba, M.; Palomares, O. The 11S globulin Sin a 2 drom yellow mustard seeds shows IgE cross-reactivity with homologous counterparts from tree nuts and peanut. Clin. Transl. Allergy 2012, 2, 23. [CrossRef] [PubMed]

19. Polk, B.; Dinakarpandian, D.; Nanda, M.; Barnes, C.; Dinakar, C. Association of tree nut and coconut sensitizations. Ann. Allergy Asthma Immunol. 2016, 117, 412-416. [CrossRef]

20. Dubiela, P.; Kabasser, S.; Smargiasso, N.; Geiselhart, S.; Bublin, M.; Hafner, C.; Mazzucchelli, G.; Hoffmann-Sommergruber, K. Jug r 6 is the allergenic vicilin present in walnut responsible for $\operatorname{IgE}$ cross-reactivity to other three nuts and seeds. Sci. Rep. 2018, 8, 11366. [CrossRef]

21. Too, J.J.Y.; Shek, L.P.C.; Rajakulendran, M. Cross-reactivity of pink peppercorn in cashew and pistachio allergic individuals. Asia Pac. Allergy 2019, 9, e25. [CrossRef] [PubMed]

22. Azcona, O.M.; Romero, L.; Bartolome, B.; Balboa, V.; Vila, L. Clinical cross-reactivity among mango, pistachio nut, and cashew nut in allergic children. J. Allergy Clin. Immunol. Pract. 2020, 8, 3234-3236.e2. [CrossRef] [PubMed]

23. Agne, P.S.; Bidat, E.; Agne, P.S.; Rancé, F.; Paty, E. Sesame seed allergy in children. Eur. Ann. Allergy Clin. Immunol. 2004, 36, 300-305. [PubMed]

24. Smith, P.K.; Krohn, R.I.; Hermanson, G.T.; Mallia, A.K.; Gartner, F.H.; Provenzano, M.D.; Fujimoto, E.K.; Goeke, N.M.; Olson, B.J.; Klenk, D.C. Measurement of protein using bicinchoninic acid. Anal. Biochem. 1987, 150, 76-85. [CrossRef]

25. Tastet, C.; Lescuyer, P.; Diemer, H.; Luche, S.; van Dorsselaer, A.; Rabilloud, T. A versatile electrophoresis system for the analysis of high- and low-molecular-weight proteins. Electrophoresis 2003, 24, 1787-1794. [CrossRef] [PubMed]

26. Shevchenko, A.; Wilm, M.; Vorm, O.; Jensen, O.N.; Podtelejnikov, A.V.; Neubauer, G.; Shevchenko, A.; Mortensen, P.; Mann, M. A strategy for identifying gel-separated proteins in sequence databases by MS alone. Biochem. Soc. Trans. 1996, 24, 893-896. [CrossRef]

27. Frank, R. Spot-synthesis: An easy technique for the positionally addressable parallel chemical synthesis on a membrane support. Tetrahedron 1992, 48, 9217-9232. [CrossRef]

28. Laune, D.; Molina, F.; Ferrieres, G.; Villard, S.; Bes, C.; Rieunier, F.; Chardes, T.; Granier, C. Application of the Spot method to the identification of peptides and amino acids from the antibody paratope that contribute to antigen binding. J. Immunol. Methods 2002, 267, 53-70. [CrossRef]

29. Notredame, C.; Higgins, D.G.; Heringa, J. T-coffee: A novel method for fast and accurate multiple sequence alignment. J. Mol. Biol. 2000, 302, 1331-1338. [CrossRef] 
30. Krieger, E.; Koraimann, G.; Vriend, G. Increasing the precision of comparative models with YASARA NOVA-A self-parameterizing force field. Proteins 2002, 47, 393-402. [CrossRef]

31. Berman, H.M.; Westbrook, J.; Feng, Z.; Gilliland, G.; Bhat, T.N.; Weissig, H.; Shindyalov, I.N.; Bourne, P.E. The protein data bank. Nucleic Acids Res. 2000, 28, 235-242. [CrossRef] [PubMed]

32. Ullah, A.; Barros Mariutti, R.; Massod, R.; Putinhon Caruso, I.; Gravatim Costa, G.H.; de Freita, C.M.; Santos, C.R.; Zanphorlin, L.M.; Rossini Mutton, M.J.; Murakami, M.T.; et al. Crystal structure of mature 2S albumin from Moringa oleifera seeds. Biochem. Biophys. Res. Commun. 2015, 468, 365-371. [CrossRef] [PubMed]

33. Mueller, G.A.; Gosavi, R.A.; Pomés, A.; Wünschmann, S.; Moon, A.F.; London, R.E.; Pedersen, L.C. Ara h 2: Crystal structure and IgE binding distinguish two subpopulations of peanut allergic patients by epitope diversity. Allergy 2011, 66, 878-885. [CrossRef]

34. Rundqvist, L.; Tengel, T.; Zdunek, J.; Björn, E.; Schleucher, J.; Alcocer, M.J.C.; Larsson, G. Solution structure, copper binding and backbone dynamics of recombinant Ber e 1-The major allergen from Brazil nut. PLOS ONE 2012, 7, e46435. [CrossRef] [PubMed]

35. Li, D.-F.; Jiang, P.; Zhu, D.-Y.; Hu, Y.; Max, M.; Wang, D.C. Crystal structure of mabinlin II: A novel structural type of sweet proteins and the main structural basis for its sweetness. J. Struct. Biol. 2008, 162, 50-62. [CrossRef]

36. Jain, A.; Kumar, A.; Salunke, D.M. Crystal structure of the vicilin from Solanum melongena reveals existence of different anionic ligands in structurally similar pockets. Sci. Rep. 2016, 6, 23600. [CrossRef]

37. Wlodawer, A.; Dauter, Z.; Porebski, P.; Minor, W.; Stanfield, R.; Jaskolski, M.; Pozharski, E.; Weichenberger, C.X.; Rupp, B. Detect, correct, retract: How to manage incorrect structural models. FEBS J. 2018, 285, 444-466. [CrossRef] [PubMed]

38. Shikhi, M.; Nair, D.T.; Salunke, D.M. Structure-guided identification of function: Role of Capsicum annuum vicilin during oxidative stress. Biochem. J. 2018, 475, 3057-3071. [CrossRef]

39. Zhang, Y.; Lee, B.; Du, W.-X.; Zhang, Y.; Wang, S.; Fan, Y.; Yi, J.; McHugh, T.H. Identification and characterization of a new Pecan [Carya illinoinensis (Wangenh.) K. Koch] allergen, Car i 2. J. Agric. Food Chem. 2016, 64, 4146-4151. [CrossRef]

40. Jin, T.; Wang, Y.; Chen, Y.-W.; Fu, T.-J.; Kothary, M.H.; McHugh, T.H.; Zhang, Y. Crystal structure of Korean pine (Pinus koraiensis) 7S seed storage protein with copper ligands. J. Agric. Food Chem. 2014, 62, 222-228. [CrossRef] [PubMed]

41. Kumar, P.; Kesari, P.; Dhindwal, S.; Choudhary, A.K.; Katiki, M.; Verma, A.; Ambatipudi, K.; Tomar, S.; Sharma, A.K.; Mishra, G.; et al. A novel function for globulin in sequestering plant hormone: Crystal structure of Wirightia tinctoria 11S globulin in coimplex with auxin. Sci. Rep. 2017, 7, 4705. [CrossRef]

42. Jin, T.; Wang, C.; Zhang, C.; Wang, Y.; Chen, Y.-W.; Guo, F.; Howard, A.; Cao, M.-J.; Fu, T.-J.; McHugh, T.H.; et al. Crystal structure of cocosin, a potential food allergen from coconut (Cocos nucifera). J. Agric. Food Chem. 2017, 65, 7560-7568. [CrossRef]

43. Tandang-Silvas, M.R.G.; Fukuda, T.; Fukuda, C.; Prak, K.; Cabanos, C.; Kimura, A.; Itoh, T.; Mikami, B.; Utsumi, S.; Maruyama, N. Conservation and divergence of plant seed $11 \mathrm{~S}$ globulins based on crystal structures. Biochim. Biophys. Acta 2010, 1804, 1432-1442. [CrossRef]

44. Jin, T.; Albillos, S.M.; Guo, F.; Howard, A.; Fu, T.-J.; Kothary, M.H.; Zhang, Y.-Z. Crystal structure of prunin-1, a major component of the almond (Prunus dulcis) allergen amandin. J. Agric. Food Chem. 2009, 57, 8643-8651. [CrossRef]

45. Oda, Y.; Matsunaga, T.; Fukuyama, K.; Miyazaki, T.; Morimoto, T. Tertiary and quaternary structures of $0.19 \alpha$-amylase inhibitor from wheat kernel determined by $\mathrm{X}$-ray analysis at 2.06 A resolution. Biochemistry 1997, 36, 13503-13511. [CrossRef] [PubMed]

46. Lehmann, K.; Schweimer, K.; Reese, G.; Randow, S.; Suhr, M.; Becker, W.-M.; Vieths, S.; Rösch, P. Structure and stability of $2 \mathrm{~S}$ albumin-type peanut allergens: Implications for the severity of peanut allergic reactions. Biochem. J. 2006, 395, 463-472. [CrossRef]

47. Jin, T.; Guo, F.; Chen, Y.-W.; Howard, A.; Zhang, Y.-Z. Crystal structure of Ara h 3, a major allergen in peanut. Mol. Immunol. 2009, 46, 1796-1804. [CrossRef] [PubMed]

48. Laskowski, R.A.; MacArthur, M.W.; Moss, D.S.; Thornton, J.M. PROCHECK: A program to check the stereochemistry of protein structures. J. Appl. Cryst. 1993, 26, 283-291. [CrossRef] 
49. Melo, F.; Feytmans, E. Assessing protein structures with a non-local atomic interaction energy. J. Mol. Biol. 1998, 277, 1141-1152. [CrossRef]

50. Benkert, P.; Biasini, M.; Schwede, T. Toward the estimation of the absolute quality of individual protein structure models. Bioinformatics 2011, 27, 343-350. [CrossRef] [PubMed]

51. Arnold, K.; Bordoli, L.; Kopp, J.; Schwede, T. The SWISS-MODEL workspace: A web-based environment for protein structure homology modelling. Bioinformatics 2006, 22, 195-201. [CrossRef]

52. Pettersen, E.F.; Goddard, T.D.; Huang, C.C.; Couch, G.S.; Greenblatt, D.M.; Meng, E.C.; Ferrin, T.E. UCSF Chimera-A visualization system for exploratory research and analysis. J. Comput. Chem. 2004, 25, 1605-1612. [CrossRef] [PubMed]

53. Pomés, A.; Davies, J.M.; Gadermaier, G.; Hilger, C.; Holzhauser, T.; Lidholm, J.; Lopata, A.L.; Mueller, G.A.; Nandy, A.; Radauer, C.; et al. WHO/IUIS Allergen Nomenclature Sub-Committee. Mol. Immunol. 2018, 100, 3-13. [CrossRef]

54. Böhm, M.; Bohne-Lang, A.; Frank, M.; Loss, A.; Rojas-Macias, M.A.; Lütteke, T. Glycosciences.DB: An annotated data collection linking glycomics and proteomics data (2018 update). Nucleic Acids Res. 2019, 47, D1195-D1201. [CrossRef]

55. Sordet, C.; Culerrier, R.; Granier, C.; Didier, A.; Rougé, P. Expression of Jug r 1, the $2 \mathrm{~S}$ albumin allergen from walnut (Juglans regia), as a correctly folded and functional recombinant protein. Peptides 2009, 30, 1213-1221. [CrossRef]

56. Pastorello, E.A.; Varin, E.; Farioli, L.; Pravettoni, V.; Ortolani, C.; Trambaioli, C.; Fortunato, D.; Giuffrida, M.G.; Rivolta, F.; Robino, A.; et al. The major allergen of sesame seeds (Sesamum indicum) is a $2 \mathrm{~S}$ albumin. J. Chromatogr. B Biomed. Sci. Appl. 2001, 756, 85-93. [CrossRef]

57. Rougé, P.; Brunet, E.; Borges, J.P.; Jauneau, A.; Saggio, B.; Bourrier, T.; Rancé, F.; Didier, A.; Barre, A. Proteins with cupin motif as major seed allergens. Rev. Fr. Allergol. 2011, 51, 36-40. [CrossRef]

58. Aalberse, R.C.; Mueller, G.A.; Derksen, N.I.L.; Aalberse, J.A.; Edwards, L.L.; Pomés, A.; Lidholm, J.; Rispens, T.; Briza, P. Identification of the amino acid fragment of Ara h 1 as a major target of the IgE-binding activity in the basic peanut protein fraction. Clin. Exp. Allergy 2020, 50, 401-405. [CrossRef]

59. Burks, A.W.; Shin, D.; Cickrell, G.; Stanley, J.S.; Helm, R.M.; Bannon, G.A. Mapping and putational analysis of the IgE-binding epitopes on Ara h 1, a legume vicilin protein and a major allergen in peanut hypersensitivity. Eur. J. Biochem. 1997, 245, 334-339. [CrossRef]

60. Shin, D.S.; Compadre, C.M.; Maleki, S.J.; Kopper, R.A.; Sampson, H.; Huang, S.K.; Burks, A.W.; Bannon, G.A. Biochemical and structural analysis of the IgE binding sites on Ara $\mathrm{h} 1$, an abundant and highly allergic peanut protein. J. Biol. Chem. 1998, 273, 13753-13759. [CrossRef] [PubMed]

61. Nesbit, J.B.; Dchein, C.H.; Braun, B.A.; Gipson, S.A.Y.; Cheng, H.; Hurlburt, B.K.; Maleki, S.J. Epitopes with similar physicochemical properties contribute to cross reactivity between peanut and tree nuts. Mol. Immunol. 2020, 122, 223-231. [CrossRef] [PubMed]

62. Archila, L.D.; Chow, I.-T.; McGinty, J.W.; Renand, A.; Jeong, D.; Robinson, D.; Farrington, M.L.; Kwok, W.W. Ana o 1 and Ana o 2 cashew allergens share cross-reactive CD4(+) T cell epitopes with other tree nuts. Clin. Exp. Allergy 2016, 46, 871-883. [CrossRef] [PubMed]

63. Goetz, D.W.; Whisman, B.A.; Goetz, A.D. Cross-reactivity among edible nuts: Double immunodiffusion, crossed immunoelectrophoresis, and human specific IgE serologic surveys. Ann. Allergy Asthma Immunol. 2005, 95, 45-52. [CrossRef]

64. De Leon, M.P.; Glaspole, I.N.; Drew, A.C.; Rolland, J.M.; O’Hehir, R.E.; Suphioglu, C. Immunological analysis of allergenic cross-reactivity between peanut and tree nuts. Clin. Exp. Allergy 2003, 33, 1273-1280. [CrossRef]

65. De Leon, M.P.; Drew, A.C.; Glaspole, I.N.; Suphioglu, C.; Rolland, J.M.; O’Hehir, R.E. Functional analysis of cross-reactive immunoglobulin E antibodies: Peanut-specific immunoglobulin E sensitizes basophils to tree nut allergens. Clin. Exp. Allergy 2005, 35, 1056-1064. [CrossRef] [PubMed]

66. Maleki, S.J.; Kopper, R.A.; Shin, D.S.; Park, C.-W.; Compadre, C.M.; Sampson, H.A.; Burks, A.W.; Bannon, G.A. Structure of the major peanut allergen Ara $\mathrm{h} 1$ may protect IgE-binding epitopes from degradation. J. Immunol. 2000, 164, 5844-5849. [CrossRef]

67. Robotham, J.M.; Wang, F.; Seamon, V.; Teuber, S.S.; Sathe, S.K.; Sampson, H.A.; Beyer, K.; Seavy, M.; Roux, K.H. Ana o 3, an important cashew nut (Anacardium occidentale L.) allergen of the $2 \mathrm{~S}$ albumin family. J. Allergy Clin. Immunol. 2005, 115, 1284-1290. [CrossRef] [PubMed] 
68. Xia, L.; Willison, L.N.; Porter, L.; Robotham, J.M.; Teuber, S.S.; Sathe, S.K.; Roux, K.H. Mapping of a conformational epitope on the cashew allergen Ana o 2: A discontinuous large sub-unit epitope dependent upon homologous or heterologous small subunit association. Mol. Immunol. 2010, 47, 1808-1816. [CrossRef]

69. Robotham, J.M.; Xia, L.; Willison, L.N.; Teuber, S.S.; Sathe, S.K.; Roux, K.H. Characterization of a cashew allergen, 11S globulin (Ana o 2), conformational epitope. Mol. Immunol. 2010, 47, 1830-1838. [CrossRef]

70. Van Ree, R.; Cabanes-Macheteau, M.; Akkerdaast, J.; Milazzo, J.-P.; Loutelier-Bourhis, C.; Rayon, C.; Villalba, M.; Koppelman, S.; Aalberse, R.; Rodriguez, R.; et al. $\beta(1,2)$-xylose and $\alpha(1,3)$-fucose residues have a strong contribution in IgE binding to plant glycoallergens. J. Biol. Chem. 2000, 275, 11451-11458. [CrossRef]

71. Andorf, S.; Borres, M.P.; Block, W.; Tupa, D.; Bollyky, J.B.; Sampath, V.; Elizur, A.; Lidholm, J.; Jones, J.E.; Galli, S.J.; et al. Association of clinical reactivity with sensitization to allergen components in multifood-allergic children. J. Allergy. Clin. Immunol. Pract. 2017, 5, 1325-1334. [CrossRef] [PubMed]

72. Van derValk, J.P.M.; El Bouche, R.; Gerth van Wijk, R.; de Groot, H.; Wichers, H.J.; Dubois, A.E.J.; de Jong, N.W. Low percentage of clinically relevant pistachio nut and mango co-sensitisation in cashew nut sensitised children. Clin. Transl. Allergy 2017, 7, 8. [CrossRef]

73. Couch, C.; Franxman, T.; Greenhawt, M. Characteristics of tree nut challenges in tree nut allergic and tree nut sensitized individuals. Ann. Allergy Asthma Immunol. 2017, 118, 591-596.e3. [CrossRef] [PubMed]

74. Fong, A.T.; Du Toit, G.; Versteeg, S.A.; van Ree, R. Pink peppercorn: A cross-reactive risk for cashew- and pistachio-allergic patients. J. Allergy Clin. Immuinol. Pract. 2019, 7, 724-725.e1. [CrossRef]

75. Cetinkaya, P.G.; Karaguzei, D.; Esenboga, S.; Sahiner, U.M.; Soyer, O.; Buyuktiryaki, B.; Birben, E.; Karaaslan, C.; Sekerel, B.E. Pistachio and cashew nut allergy in childhood: Predictive factors towards development of a decision tree. Asian Pac. J. Allergy Immunol. 2019. [CrossRef]

76. Amat, F.; Benharoun-Stern, R.; Benissa, M.-R.; Just, J. Usefulness of rAna o 3 assessment before oral food challenge to pistachio. Pediatr. Allergy Immunol. 2020. [Online ahead of print]. [CrossRef]

77. Cetinkaya, P.G.; Buyuktiryaki, B.; Soyer, O.; Sahiner, U.M.; Sackesen, C.; Sekerel, B.E. Phenotypical characterization of tree nuts and peanut allergies in esat Mediterranean children. Allergol. Immunopathol. 2020, 48, 316-322. [CrossRef]

78. Saba, L.; Clerc-Urmès, I.; Delahaye, C.; Chevillot, E.; Jarlot-Chevaux, S.; Dumond, P.; Schweitzer, C.; Divaret-Chauveau, A. Predictive factors of allergy to pistachio in children allergic to cashew nut. Pediatr. Allergy Immunol. 2020, 31, 506-514. [CrossRef]

Publisher's Note: MDPI stays neutral with regard to jurisdictional claims in published maps and institutional affiliations.

(C) 2021 by the authors. Licensee MDPI, Basel, Switzerland. This article is an open access article distributed under the terms and conditions of the Creative Commons Attribution (CC BY) license (http://creativecommons.org/licenses/by/4.0/). 